\title{
REVIEW
}

\section{A Systematic Review of Factors Associated with Non- Adherence to Treatment for Immune-Mediated Inflammatory Diseases}

\author{
Eleni Vangeli · Savita Bakhshi · Anna Baker · Abigail Fisher • \\ Delaney Bucknor · Ulrich Mrowietz · Andrew J. K. Östör · Laurent Peyrin-Biroulet • \\ Ana P. Lacerda $\cdot$ John Weinman
}

To view enhanced content go to www.advancesintherapy.com

Received: July 14, 2015 / Published online: November 7, 2015

(C) The Author(s) 2015. This article is published with open access at Springerlink.com

\begin{abstract}
Background: Non-adherence impacts negatively on patient health outcomes and has associated economic costs. Understanding drivers of treatment adherence in immune-mediated inflammatory diseases is key for the development of effective strategies to tackle non-adherence.
\end{abstract}

Electronic supplementary material The online version of this article (doi:10.1007/s12325-015-0256-7) contains supplementary material, which is available to authorized users.

E. Vangeli

Department of Psychology, London South Bank University, London, UK

S. Bakhshi

Psychological Medicine, King's College London, London, UK

A. Baker

Atlantis Healthcare, London, UK

A. Fisher

University College London, London, UK

D. Bucknor

London Metropolitan University, London, UK

U. Mrowietz

Psoriasis-Center at the Department of Dermatology, University Medical Center of Schleswig-Holstein,

Campus Kiel, Germany
Objective: To identify factors associated with treatment non-adherence across diseases in three clinical areas: rheumatology, gastroenterology, and dermatology.

Design: Systematic review.

Data Sources: Articles published in PubMed, Science Direct, PsychINFO and the Cochrane Library from January 1, 1980 to February 14, 2014.

Study Selection: Studies were eligible if they included patients with a diagnosis of rheumatoid arthritis, ankylosing spondylitis,

\section{A. J. K. Östör}

School of Clinical Medicine, University of

Cambridge, Cambridge, UK

L. Peyrin-Biroulet

Inserm U954 and Department of Gastroenterology, Université de Lorraine, Vandoeuvre-les-Nancy, France

A. P. Lacerda AbbVie Inc, North Chicago, IL, USA

J. Weinman ( $ه)$ Institute of Pharmaceutical Science, King's College London, 5th Floor, Franklin-Wilkins Building, 150 Stamford Street, London SE1 9NH, UK e-mail: John.weinman@kcl.ac.uk 
psoriatic arthritis, inflammatory bowel disease, or psoriasis and included statistics to examine associations of factors with non-adherence.

Data Extraction: Data were extracted by the first reviewer using a standardized 23-item form and verified by a second/third reviewer. Quality assessment was carried out for each study using a 16-item quality checklist.

Results: 73 studies were identified for inclusion in the review. Demographic or clinical factors were not consistently associated with non-adherence. Limited evidence was found for an association between non-adherence and treatment factors such as dosing frequency. Consistent associations with adherence were found for psychosocial factors, with the strongest evidence for the impact of the healthcare professional-patient relationship, perceptions of treatment concerns and depression, lower treatment self-efficacy and necessity beliefs, and practical barriers to treatment.

Conclusions: While examined in only a minority of studies, the strongest evidence found for non-adherence were psychosocial factors. Interventions designed to address these factors may be most effective in tackling treatment non-adherence.

Keywords: Inflammatory bowel disease; Patient adherence; Psoriasis; Psoriatic arthritis; Rheumatology

\section{INTRODUCTION}

Immune-mediated inflammatory diseases (IMIDs) refer to a group of chronic conditions that share common inflammatory pathways [1]. IMIDs include conditions such as inflammatory bowel disease (IBD), psoriasis (PS) and rheumatologic conditions (RC) including rheumatoid arthritis
(RA), ankylosing spondylitis (AS), and psoriatic arthritis (PsA). IMIDs affect approximately 5-7\% of Western populations and can have a detrimental effect on quality of life and health outcomes [1]. In line with other chronic conditions, sub-optimal adherence to treatment has been reported in a number of systematic reviews. Persistence or adherence rates to treatments for IMIDs were found to range from $30 \%$ to $80 \%$ in RA [2], $7 \%$ to $72 \%$ in IBD [3], and $33 \%$ to $78 \%$ in PS [4].

Increasing adherence may have a far greater impact on health outcomes than advances in medical treatments $[5,6]$. There are also associated economic implications such as increased medication costs, resources used including hospital admissions, inadequate use of healthcare professionals' time, and increased sickness-related work absence [7]. Thus, understanding the key drivers of non-adherence to the types of treatments used across IMIDs is an important area of investigation and key for the development of effective strategies to tackle non-adherence. Further, the identification of generic tools and/or interventions common to IMIDs would enable the identification of key areas likely to be important for adherence and assist the clinician to identify and address patient concerns in their consultations.

Although there are existing systematic reviews looking at factors associated with non-adherence in the individual clinical areas (i.e., RA, IBD, or PS), there is a clear need for a broad understanding of the determinants of adherence across IMIDs [2-4, 8-19].

\section{AIMS}

To our knowledge, no systematic review to date has examined factors associated with adherence across several IMIDs or included multiple 
treatment types. The purpose of the current review is, therefore, to examine factors associated with adherence in selected IMIDs across rheumatology, gastroenterology and dermatology in a systematic way. This could enable the identification of associations not only in each therapeutic area but also those in common across the therapeutic areas. Identification of key factors will allow interventions to focus on areas most likely to have an impact on non-adherence. If there are factors that are found to be common across these IMIDs, this will afford the opportunity to develop cross-condition tools for the health care professional (HCP) both to identify areas of non-adherence risk and for generic interventions, which may be particularly useful for rheumatologists who are likely to treat patients with different manifestations of their IMIDs.

\section{METHODS}

The systematic review followed guidelines developed by the National Institute for Clinical Excellence (NICE) and the Centre for Reviews and Dissemination, University of York $[18,19]$.

\section{Literature Search and Selection}

A search of the literature was conducted via the following online databases: PubMed, Science Direct, PsychINFO and the Cochrane Central Register of Clinical Trials. A broad search strategy was developed to capture each disease within the examined clinical areas (see Fig. 1). In addition, the reference lists of relevant articles identified through the database search and existing systematic reviews were searched manually to identify further suitable studies.
The search was limited to articles published from January 1, 1980 to February 14, 2014. The reason for limiting the search to articles published after January 1980 was that a previous systematic review identified that general research interest in treatment adherence began around 1980 [3].

The search was conducted individually for each of the selected IMIDs within the five clinical areas: RA, AS, PsA and IBD and PS. Initially, the titles and abstracts of the articles identified through the search strategies were screened by a first reviewer for eligibility (SB, AF or DB). The full text was then obtained for all shortlisted studies and independently reviewed by a second reviewer (AB). Disagreements between the two reviewers were resolved by discussion and independently assessed by a third reviewer (EV or JW).

\section{Inclusion/Exclusion Criteria}

Studies were eligible for inclusion in the review if they met all the criteria below:

- Published/in press between January 1, 1980 and February 14, 2014.

- Written in English language.

- Included patients with a diagnosis of RA, AS or PsA, IBD, or PS.

- Based primarily on adult samples ( $\geq 18$ years).

- Included statistics to examine associations of factors with non-adherence.

- Used a specified measure of adherence (validated or non-validated).

- Included adherence measurement of injection or infusion, oral, rectal or transdermal formulation (excluding parenteral nutrition).

- Contained primary quantitative data.

- All participants were on a disease-specific treatment. 


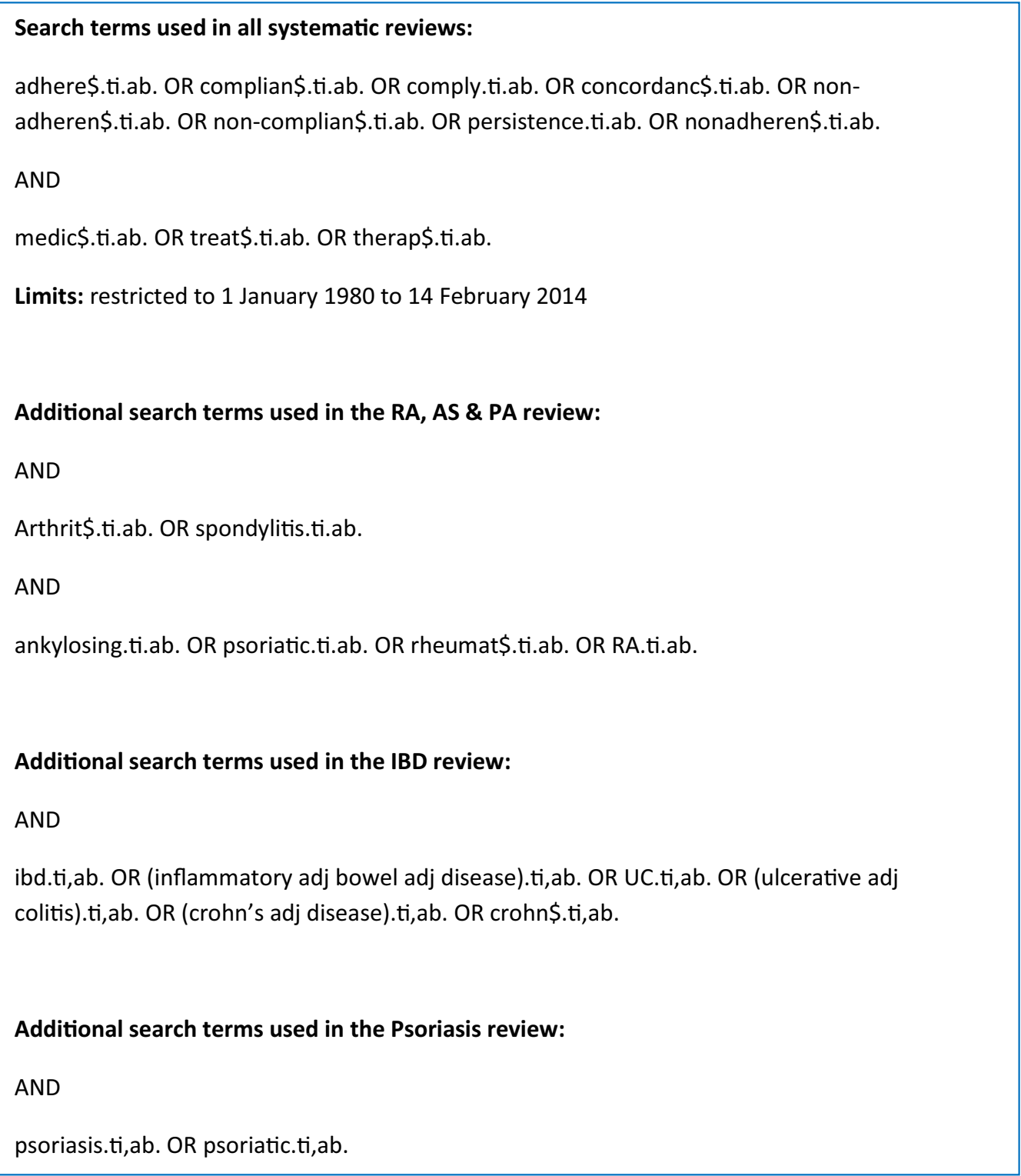

Fig. 1 Search terms

- Full study published in a peer-review journal (i.e., not a conference abstract).

Studies in other clinical indications were included as long as specific information on one of the conditions of interest was explicit within the results. The decision was taken to exclude studies examining adherence to topical treatments alone, as topical treatments are not used across all three clinical areas and are typically prescribed in mild cases of PS only.

\section{Quality Appraisal}

Quality assessment was carried out for each study to examine their susceptibility to bias in terms of rigor, methods and analysis. A 16-item quality checklist adapted from a previous systematic review of a similar nature [3] based on guidance from NICE and Strengthening the Reporting of Observational studies in Epidemiology was completed for each study. 
Although studies were not excluded or ranked according to quality, an overall quality score, based on the total number of quality criteria met, was computed for each study. Quality scores were used as general indicators for each study and are presented in the overview tables of included studies. Common quality limitations are explored in more detail in the "Results" section.

\section{Data Extraction and Synthesis}

Studies identified through each individual search were combined for data synthesis and extraction. For each eligible study, data were extracted by the first reviewer using a standardized form consisting of 23 items, which included details of measures that could potentially relate to non-adherence. Details of the sample, non-adherence measure and potential associates examined were extracted and tabulated by the first reviewer and verified by the second and third reviewers. There was an $85 \%$ initial agreement in the data extracted and all discrepancies were resolved through discussion between the reviewers.

Due to the heterogeneity of the included studies, it was not possible to perform a meta-analysis of the findings. Frequencies and proportions of studies examining similar variables and any association observed were calculated to offer a simple indication of the level of evidence. As such, the evidence was primarily synthesized in a narrative review and quantified in terms of the proportions of studies finding an association. As no two studies controlled for the same variables and the quality of these studies varied considerably, preference was not given to findings from adjusted analyses. Where associations were found for a factor and these were all in the same direction, the association was considered to be consistent.

\section{Compliance with Ethical Guidelines}

This article is based on previously conducted studies and does not involve any new studies of human or animal subjects performed by any of the authors.

\section{RESULTS}

\section{Included Studies}

A total of 73 studies met the inclusion criteria and were included in the combined review: $\mathrm{RC}=26(\mathrm{RA}=23 ; \mathrm{AS}=1 ; \mathrm{PsA}=11) ; \mathrm{IBD}=36$; $\mathrm{PS}=11$ [20-92]. Details regarding the study selection and exclusion process followed are presented in Figs. 2, 3 and 4. A summary of the characteristics of the studies and the factors examined in each study are shown in Tables 1, 2 and 3 Studies from the same authors were checked for overlapping samples, and where there was overlap in the samples, the studies examined different possible predictors of adherence $[69,70]$.

The sample size of the studies varied considerably, ranging from 28 to 12,750 participants. The vast majority of studies (90.4\%) were based on samples from Europe $(n=37,51 \%)$ or North America $(n=30,41 \%)$. Participants were derived from outpatient clinics in the majority of samples. In RC, this was $76.9 \%(n=20)$, in IBD $(n=25,69.4 \%)$ and in PS $(n=8,72.7 \%)$. One sample in RC [23] was recruited in a clinical trial and two samples in IBD $[69,79]$ were convenience samples recruited online through social media or IBD forums. The remaining samples were established cohorts drawn from medical or pharmacy databases. 


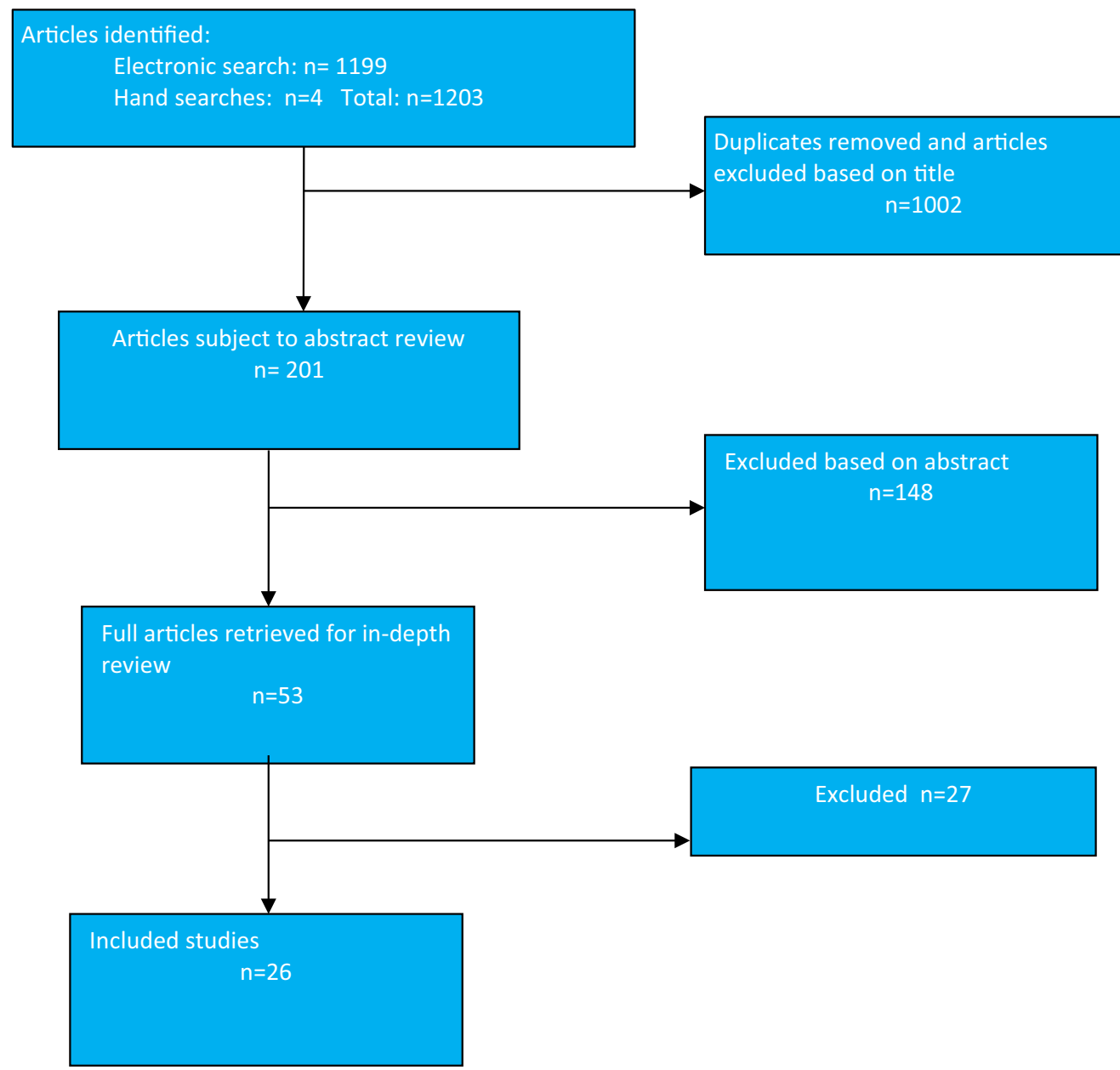

Fig. 2 Flowchart of included studies: rheumatologic conditions, reasons for exclusion of final 27 studies included: did not statistically examine factors associated with adherence $(n=8$, original search) $(n=7$, update

The proportion of longitudinal studies (including retrospective cohorts) was $57.8 \%$ $(n=15)$ in RC, $36.1 \%(n=13)$ in IBD, and $72.7 \% \quad(n=8)$ in PS. While a substantial proportion of studies had a longitudinal design, factors were most often examined as concurrent associates of adherence and not as prospective predictors. Thus, in the current review all factors are considered as potential associates of adherence.

A large proportion of studies (57.5\%) used self-report measures to assess adherence. In RC, the medication event monitoring system search), full study data not reported $(n=1$, original search), did not define measure of adherence $(n=9$, original search) ( $n=1$, update search), intervention examined in relation to adherence $(n=1$, original search)

(MEMS) was used to measure non-adherence in three studies [28, 34, 44], others used pill counts and pharmacy refill data [22, 23, 27] or plasma analysis [21]. Five studies had a measure of medication persistence (i.e., continuation with a medication) as the adherence outcome, obtained via HCP report [36, 38] or patient records/case notes $[25,26,45]$. In IBD, three studies combined self-report measurement with a biochemical measure $[39,53,59]$. One study assessed adherence using a biological measure only [58] and another via infusion appointment attendance [12]. The remaining five studies 


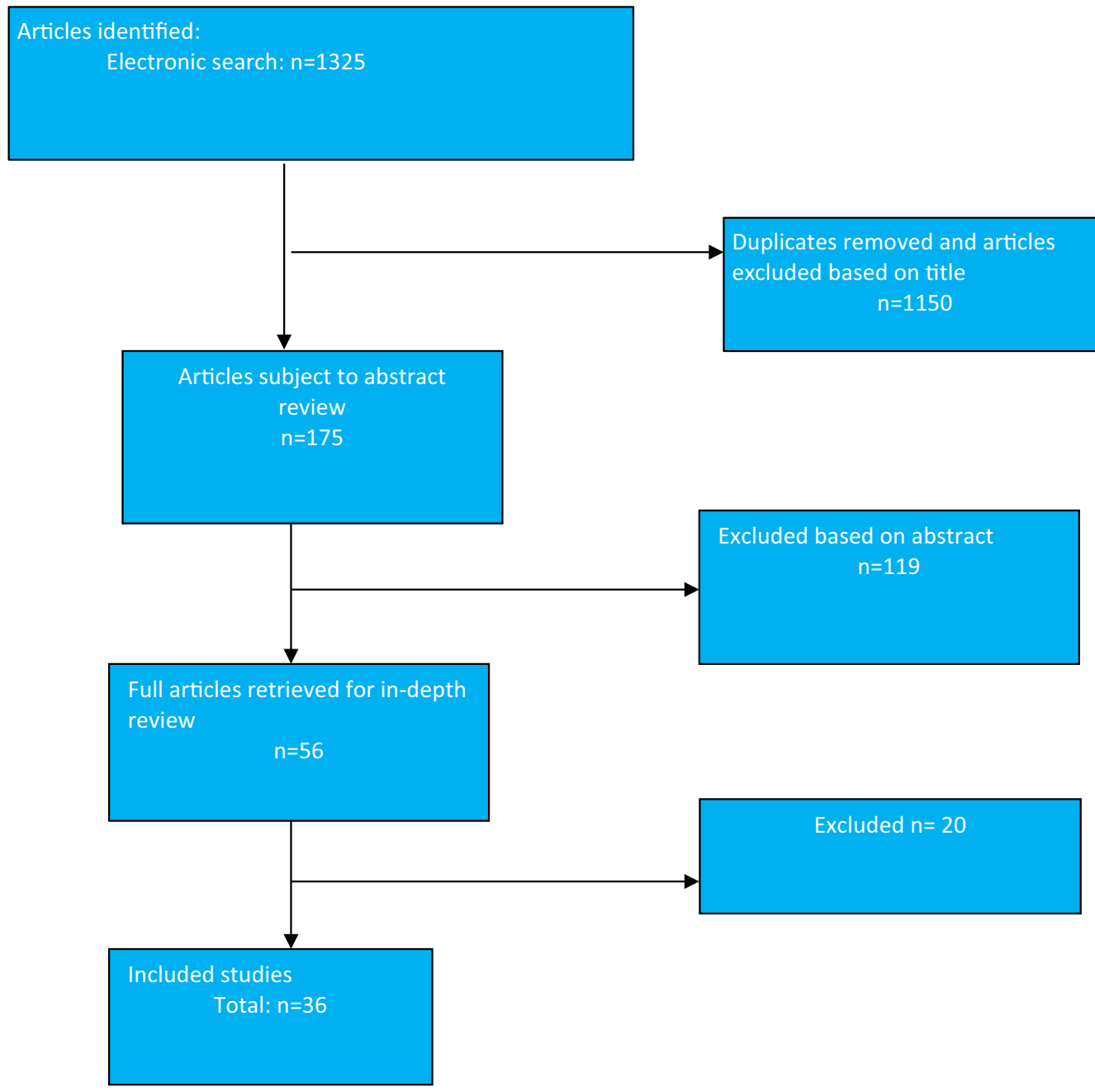

Fig. 3 Flowchart of included studies: inflammatory bowel disease, reasons for exclusion of final 20 studies included: did not statistically examine factors associated with adherence ( $n=10$, original search) ( $n=1$, update search),

used a proxy measure of adherence via prescription refill data [41, 49-51, 76]. In PS, two studies assessed adherence using a proxy measure from prescription refill data $[83,85]$. A further three studies had a measure of medication persistence as the adherence outcome obtained from patient medical records [86-88]. Two studies assessed adherence with respect to unused treatment medication ascertained via pill counts weight $[89,92]$. did not define measure of adherence $(n=5$, original search), intervention examined in relation to adherence $(n=2$, original search), adherence examined in sample of pregnant women only $(n=2$, original search)

\section{Quality of Included Studies}

The proportion of quality criteria met by each study varied widely across the three clinical areas, ranging between $31 \%$ and $87.5 \%$ in $\mathrm{RC}$, $25 \%$ and $93.8 \%$ in IBD, and $25 \%$ and $58.3 \%$ in PS. The included studies in RC typically met the highest proportion of quality criteria, whereas those in PS met the least. Quality criteria most commonly not met related to details of the study required to enable an assessment of bias. 


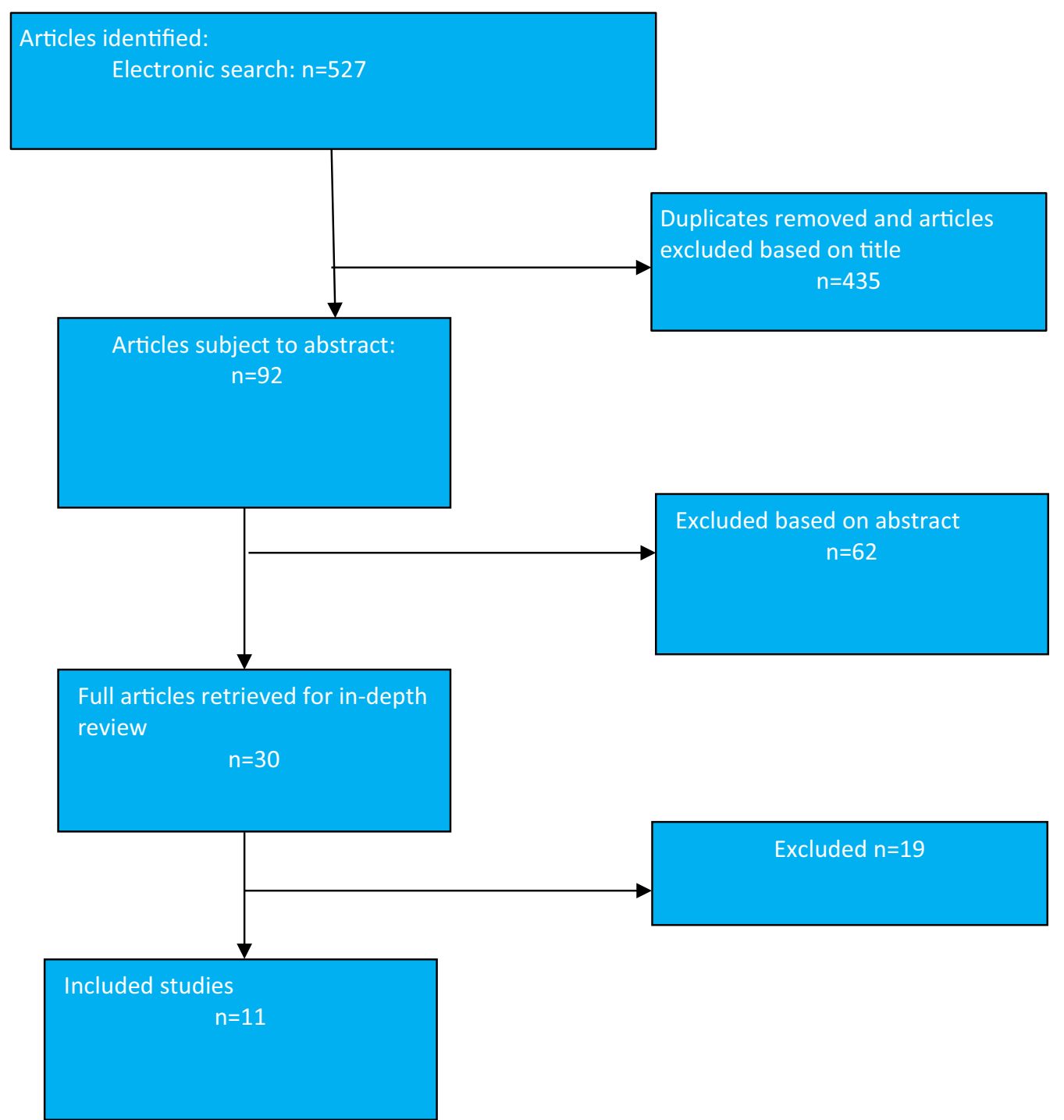

Fig. 4 Flowchart of included studies: psoriasis reasons for exclusion of final 19 studies included: did not statistically examine factors associated with adherence $(n=7$, original

A number of studies did not report details of eligibility criteria $(n=15,20.5 \%)$ or the number of participants not consenting to participate in the study $(n=42,57.5 \%)$, so it was not possible to make an assessment of biases due to participant selection. Similarly, failure to report how missing data were treated $(n=65,89 \%)$ and control for confounders $(n=35 ; 52 \%)$ was common preventing an search) ( $n=6$, update search), examined topical treatments only $(n=5$, original search), intervention examined in relation to adherence $(n=1$, original search)

assessment of the strength of the associations found. The majority of studies did not report power calculations $(n=56,77 \%)$ to estimate their sample sizes and as such it was difficult to assess whether studies were adequately powered to detect associations. However, several studies had very small sample sizes that were unlikely to result in adequate power for the statistics applied. 


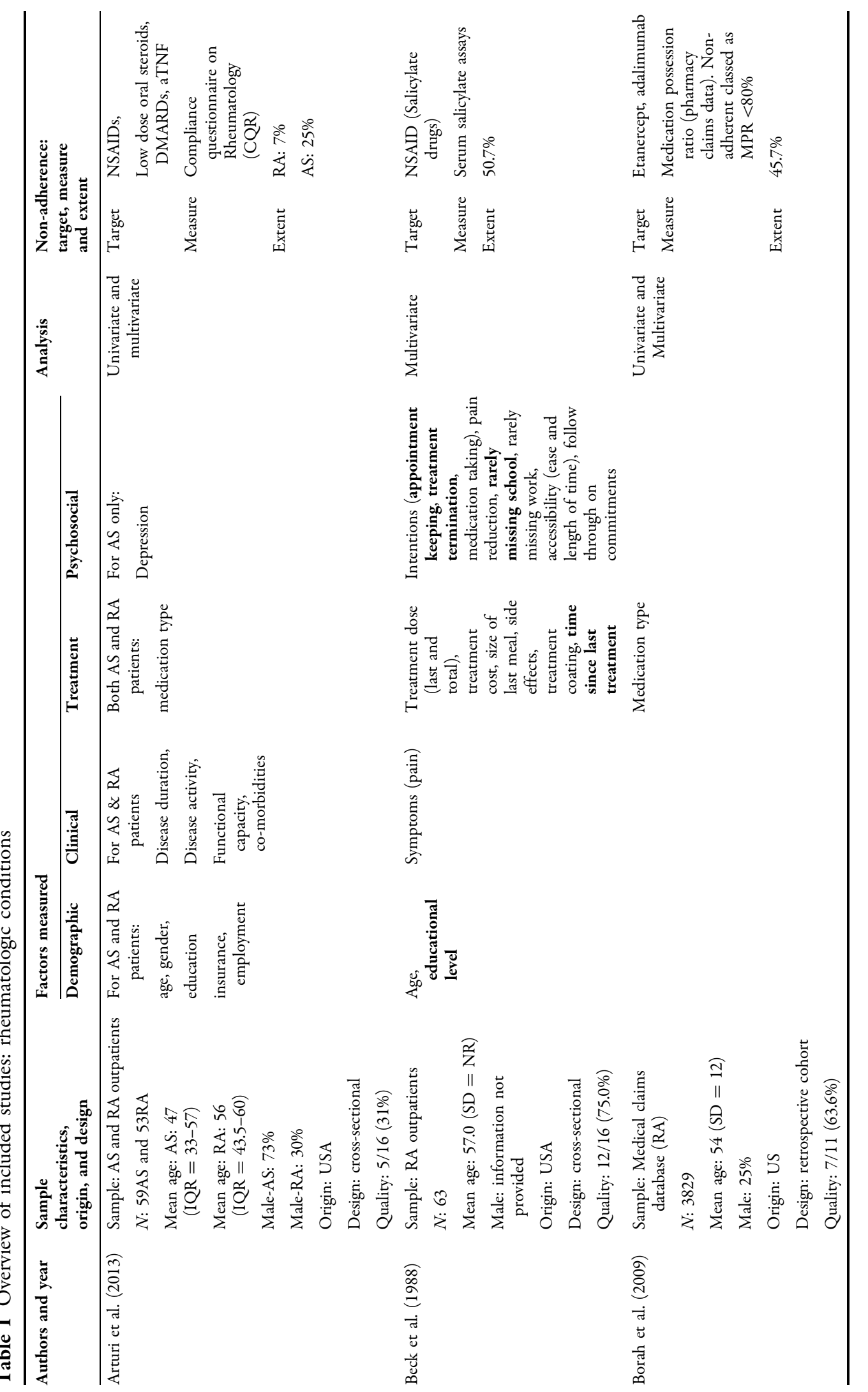




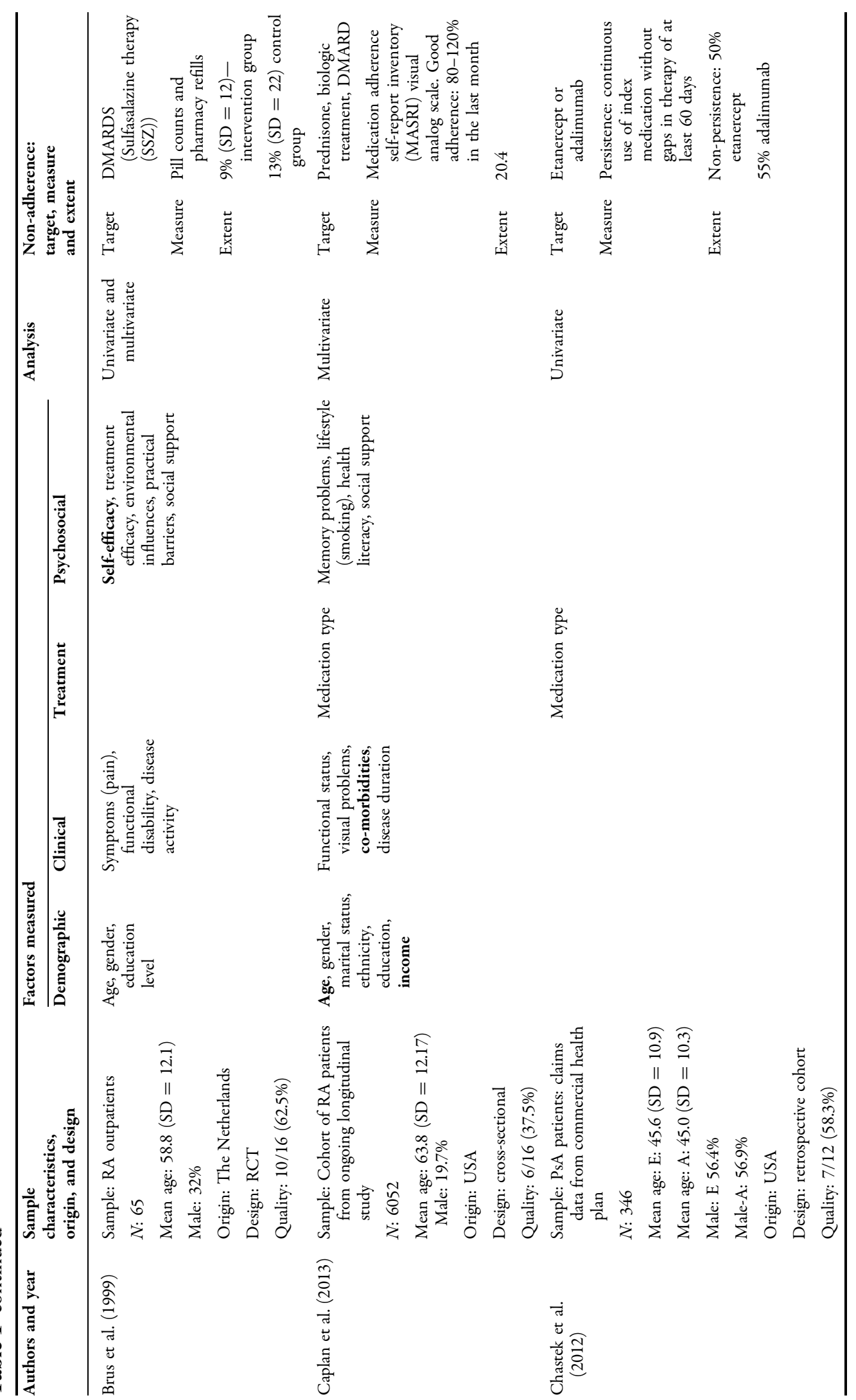




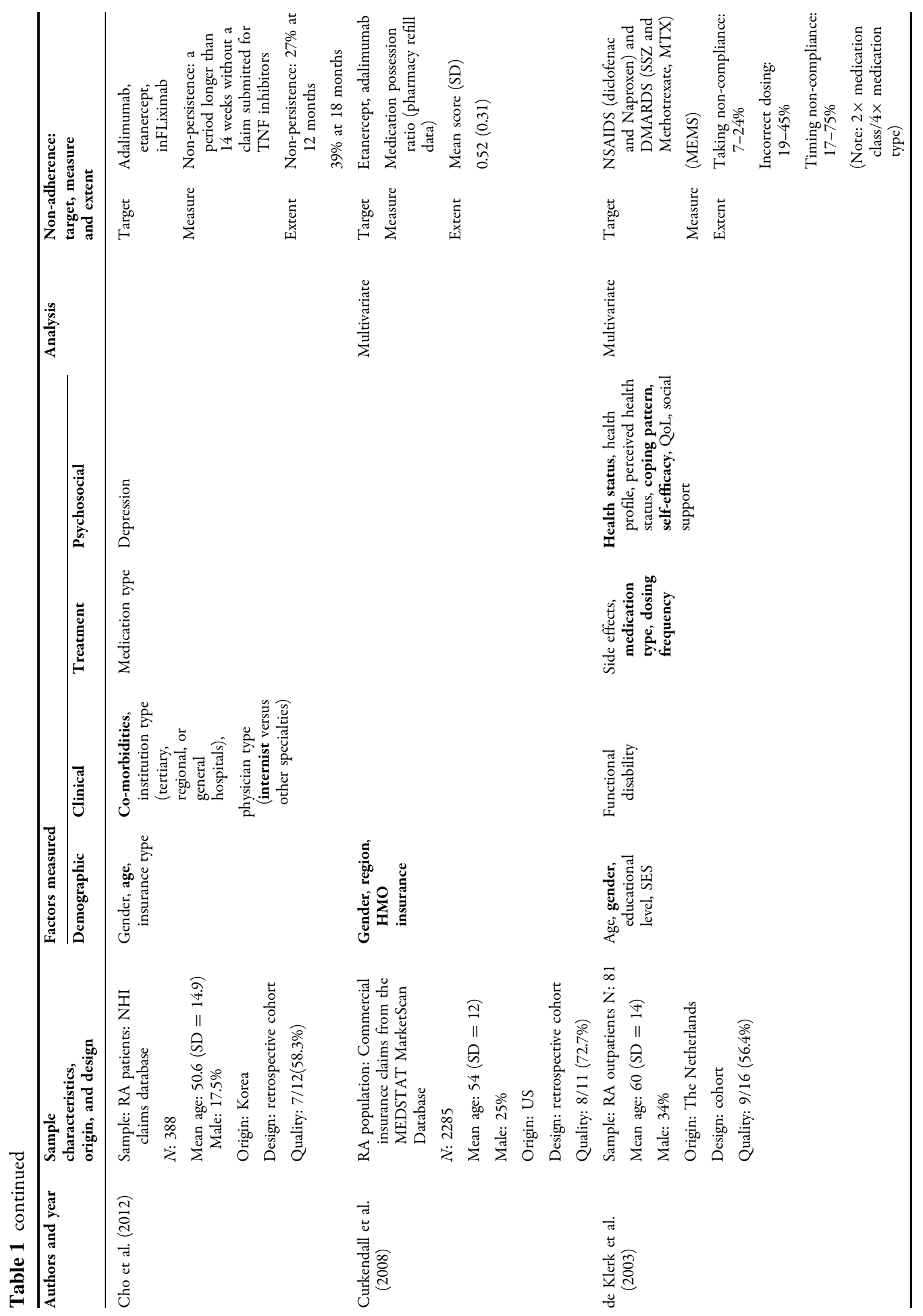




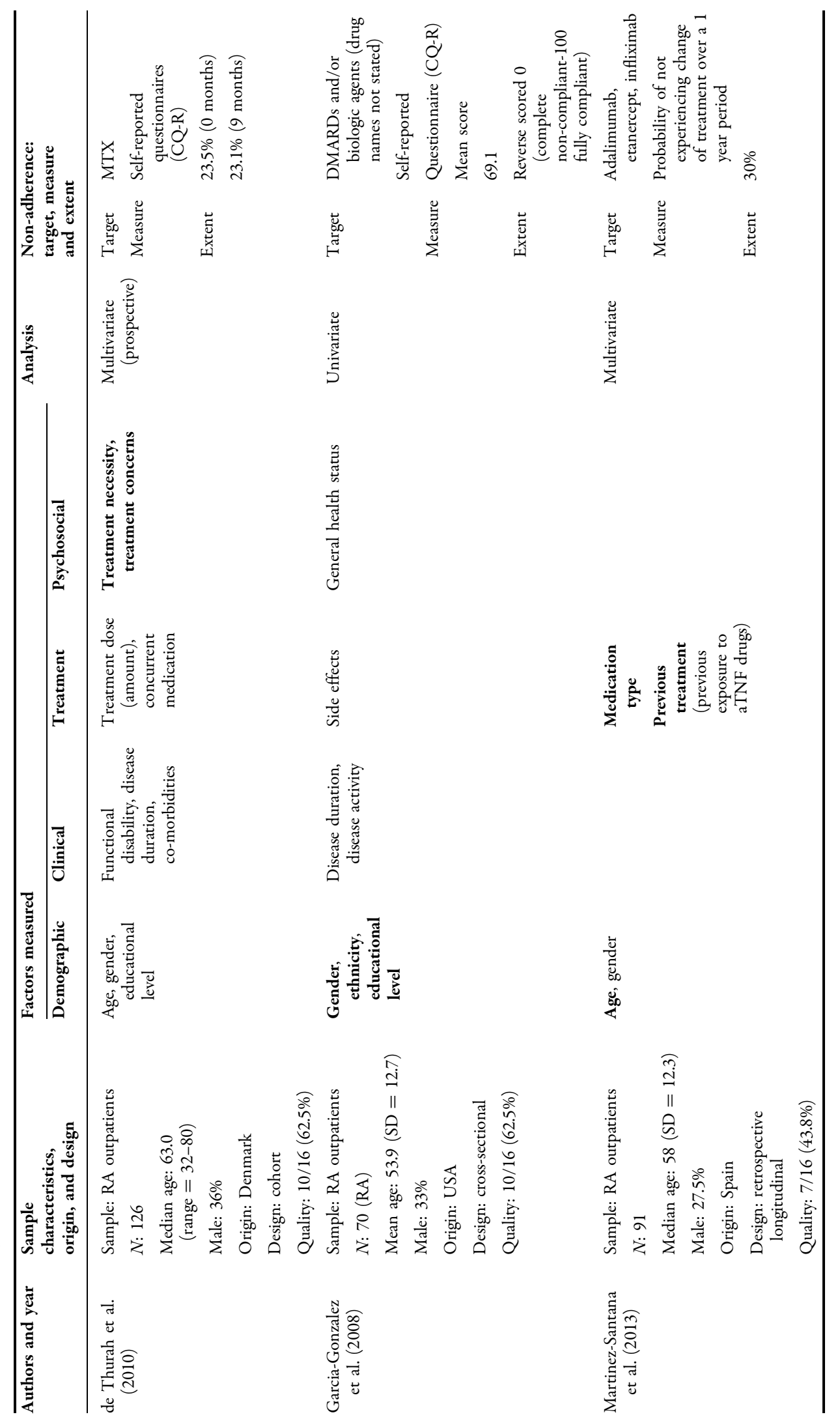




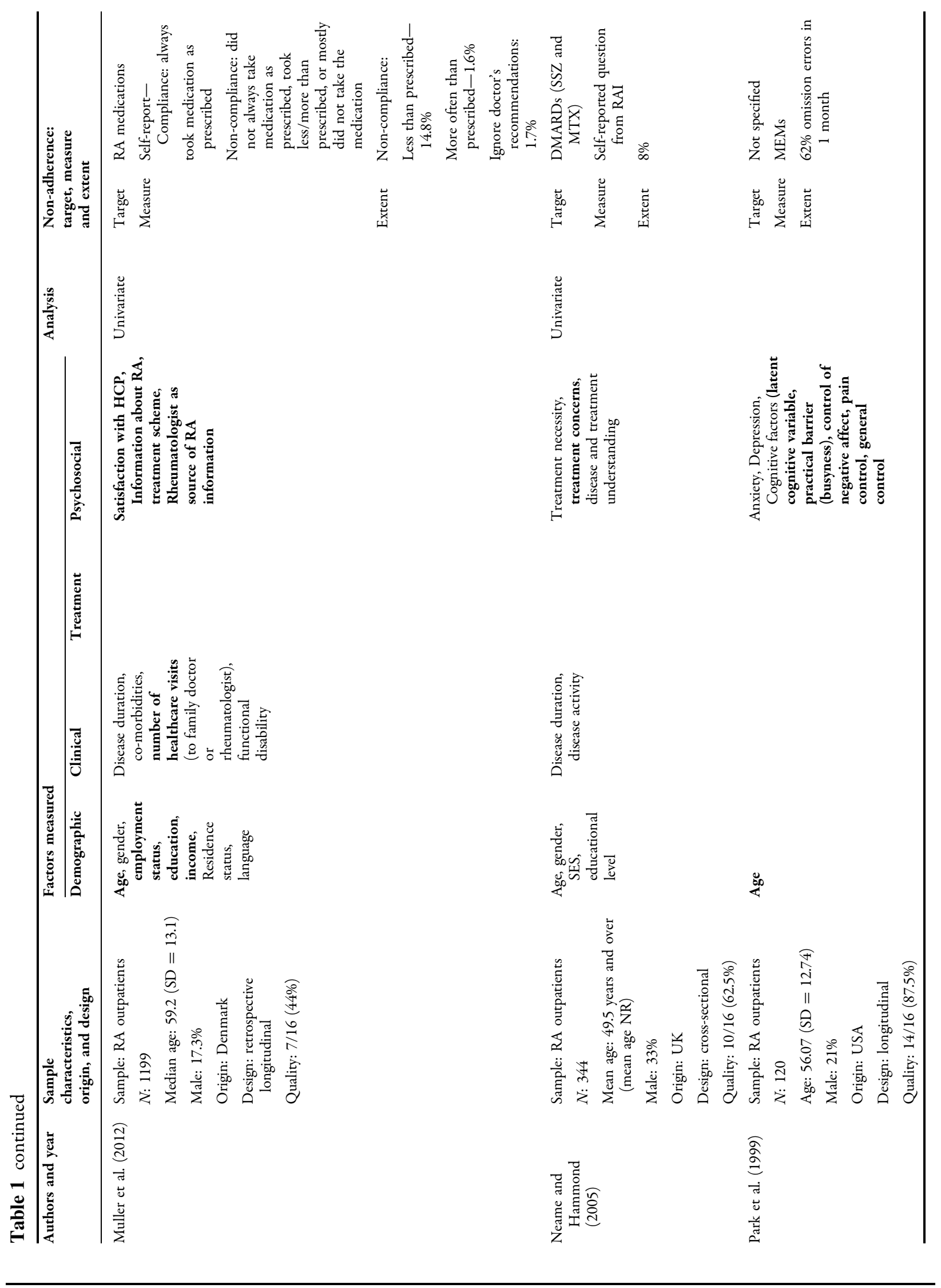




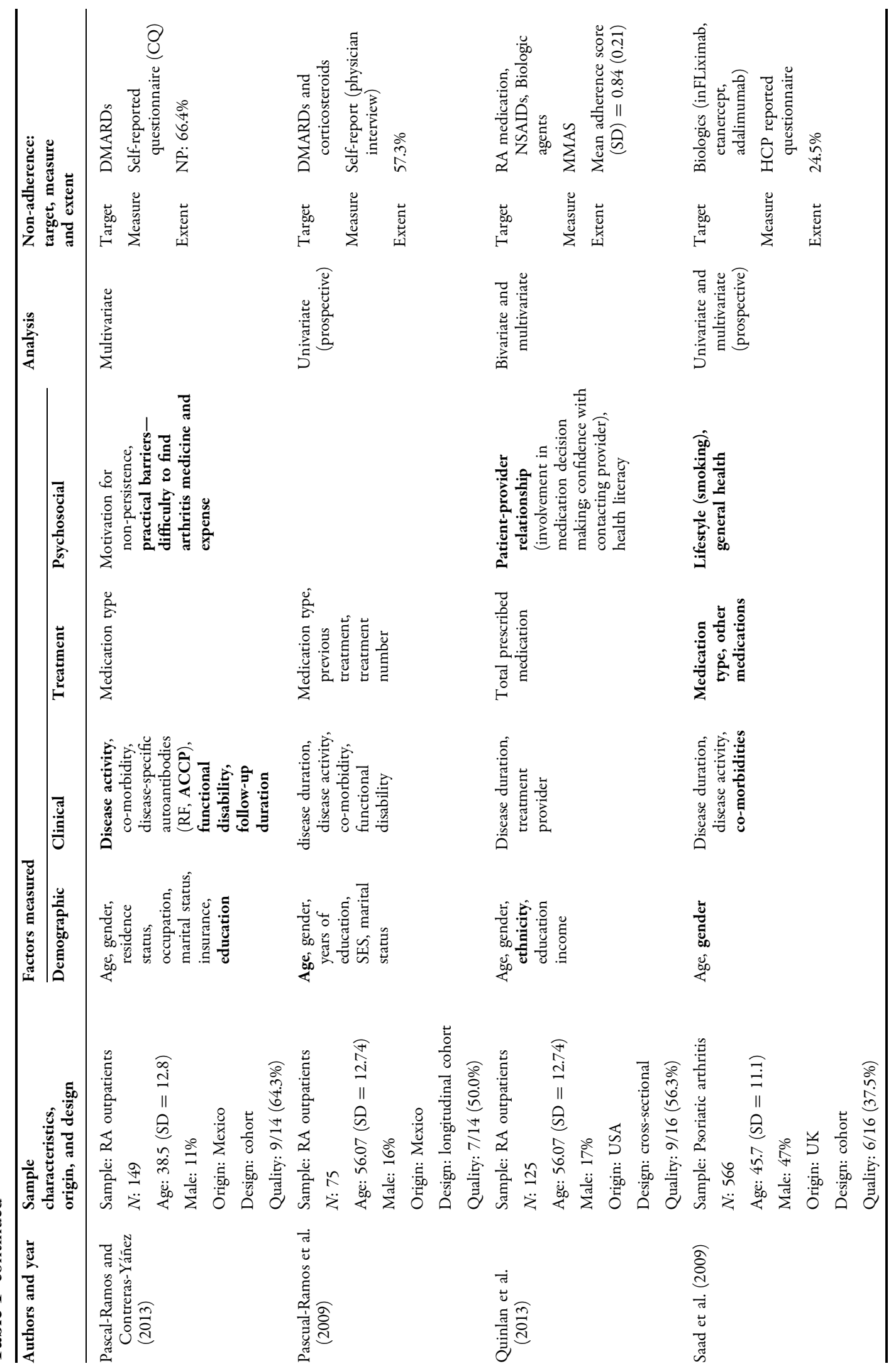




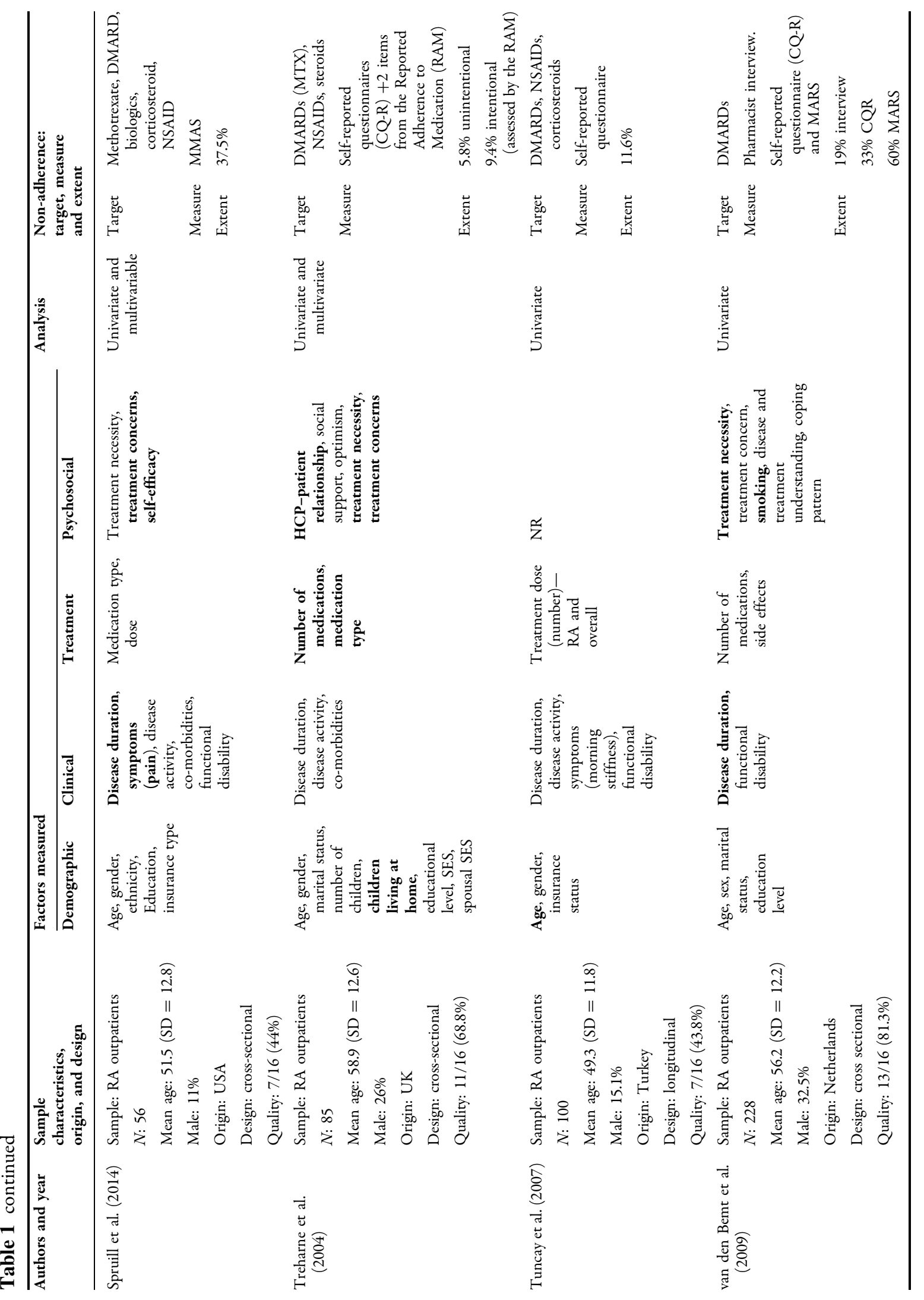




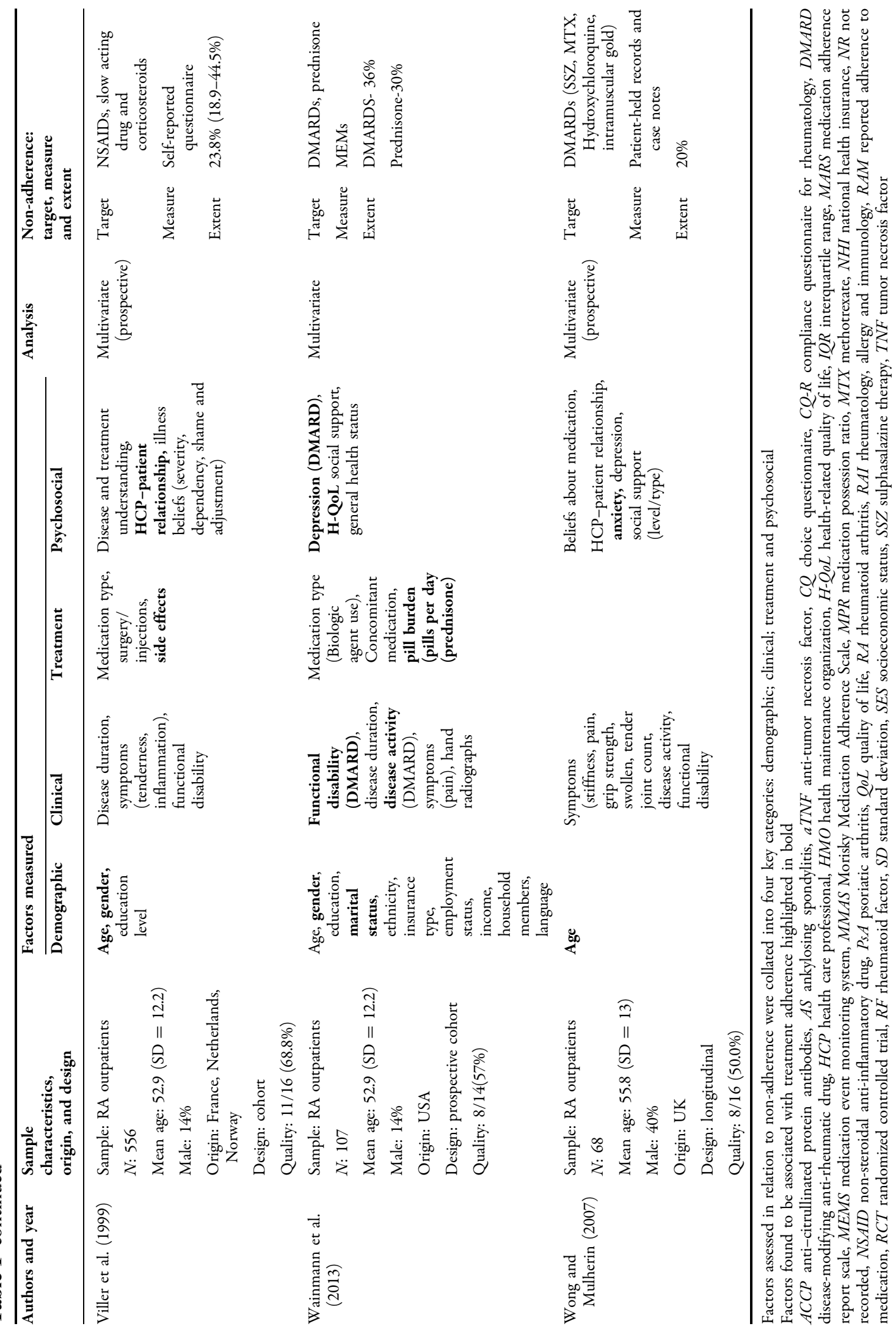




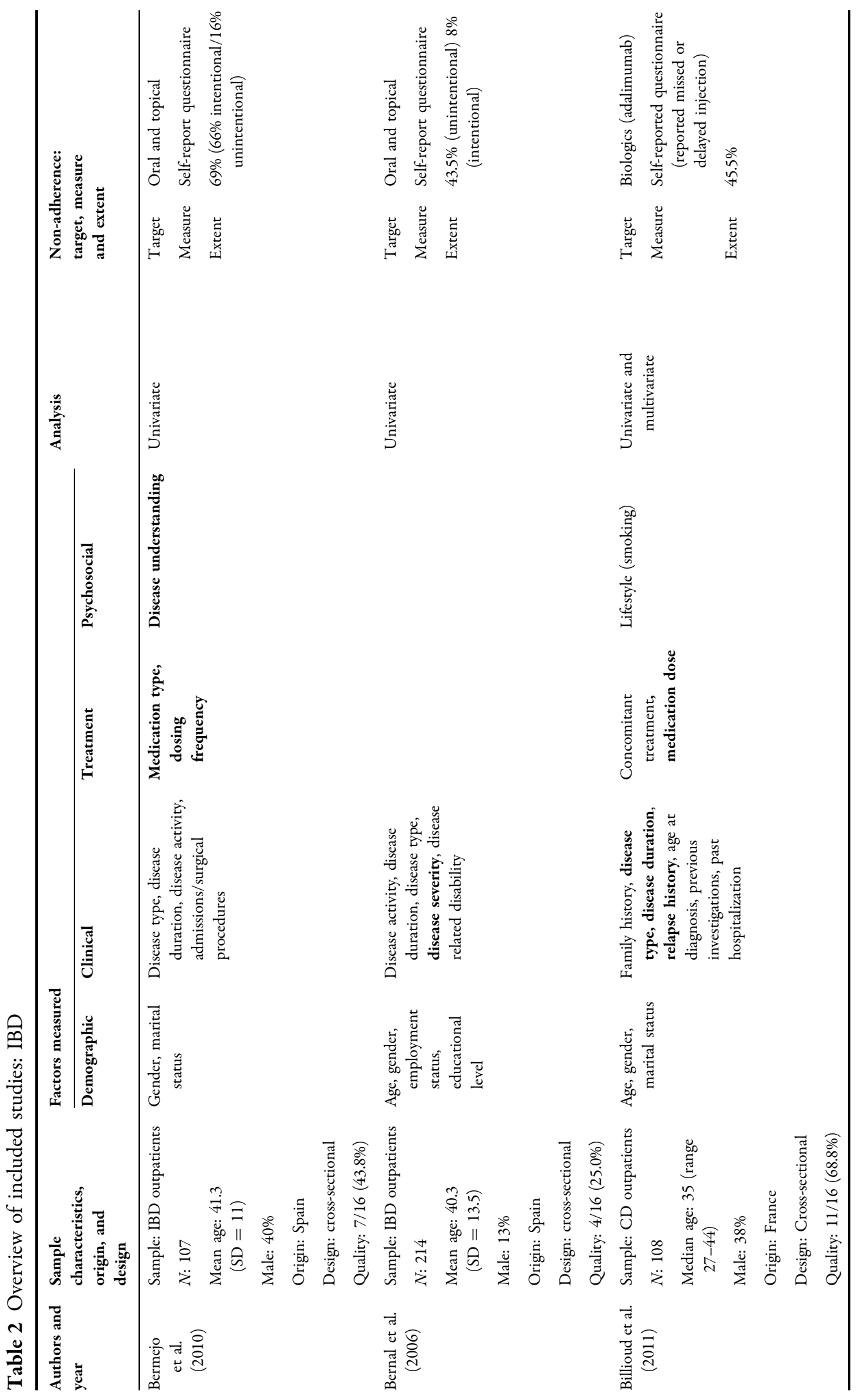




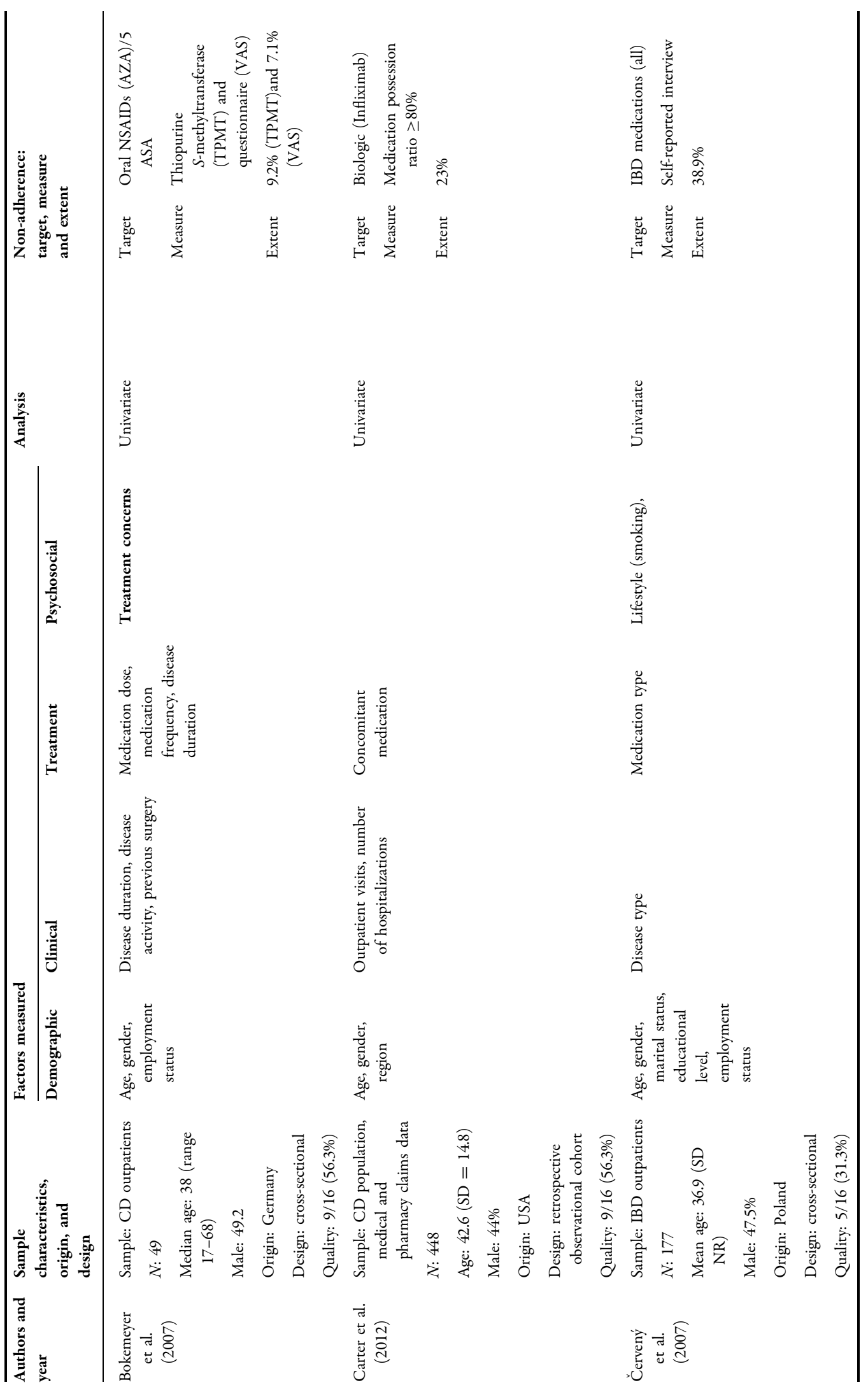




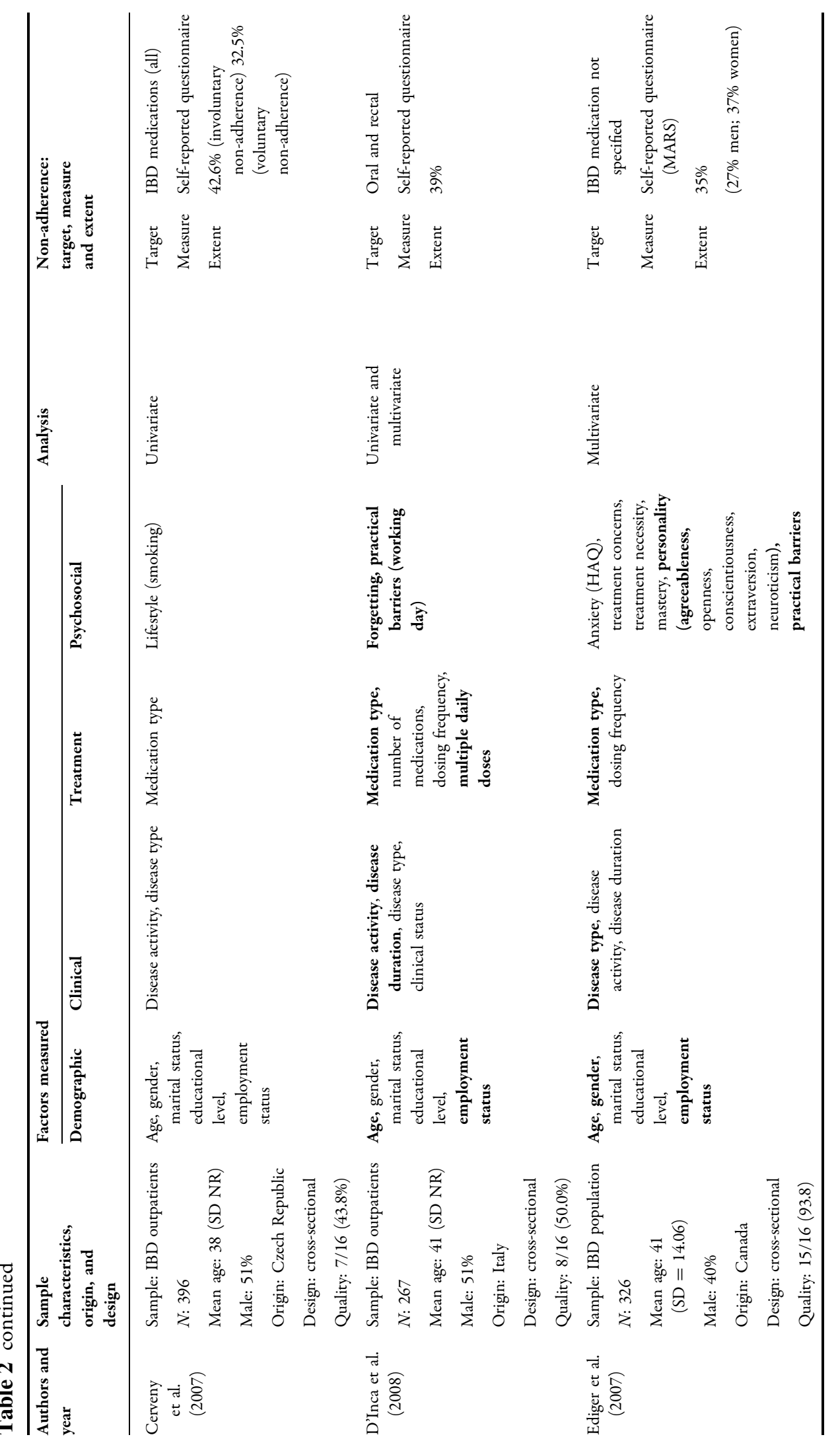




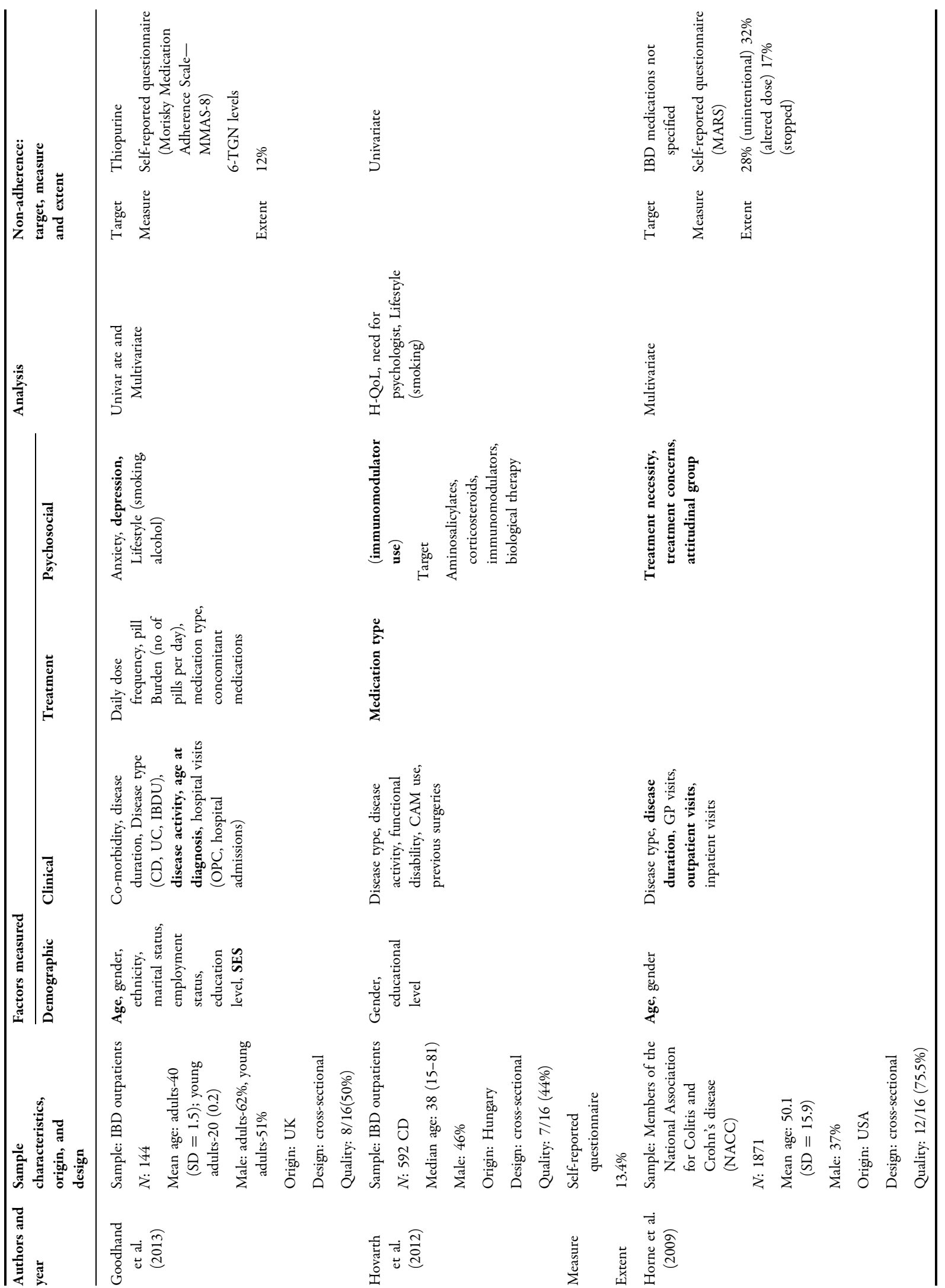




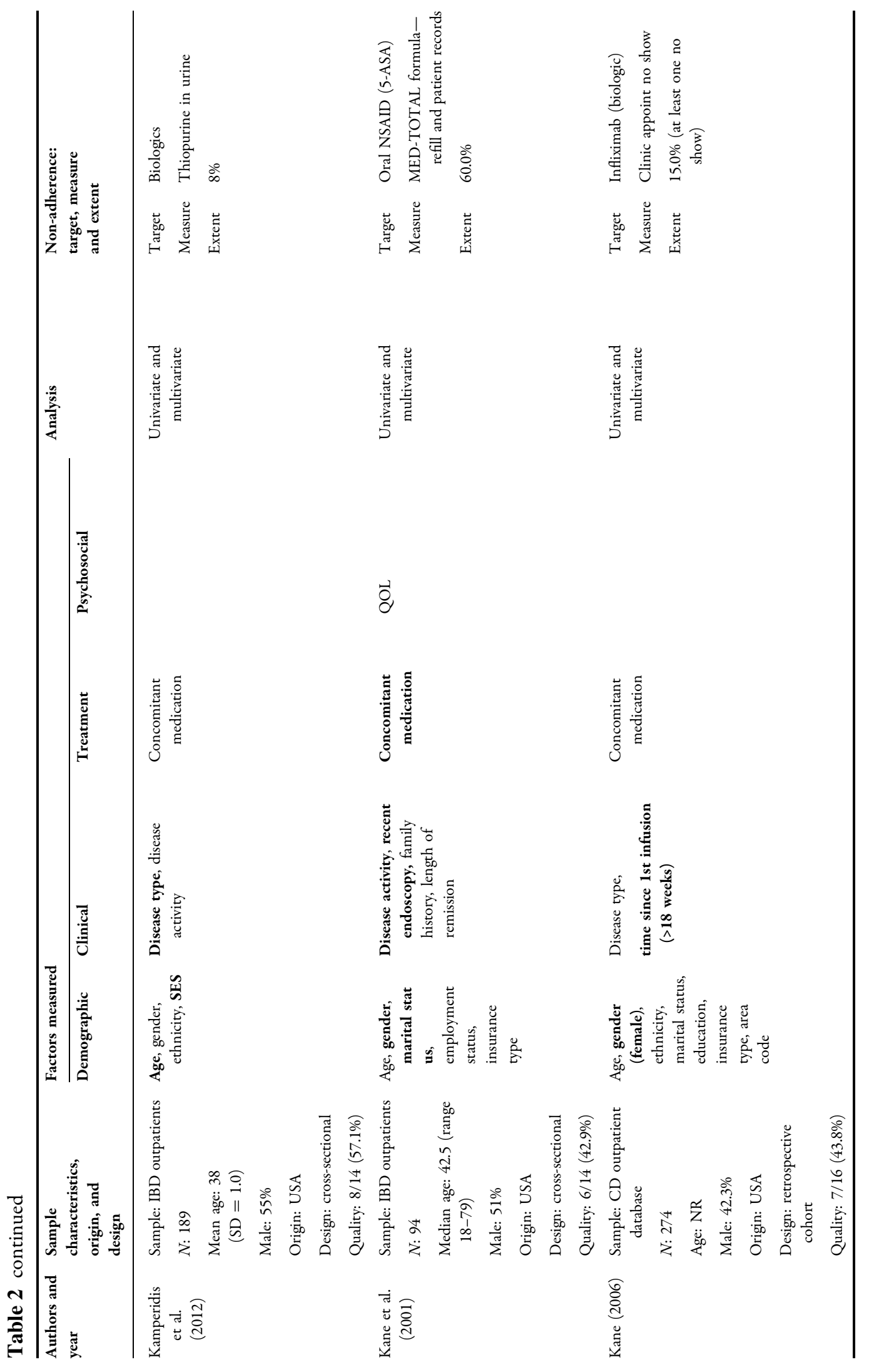




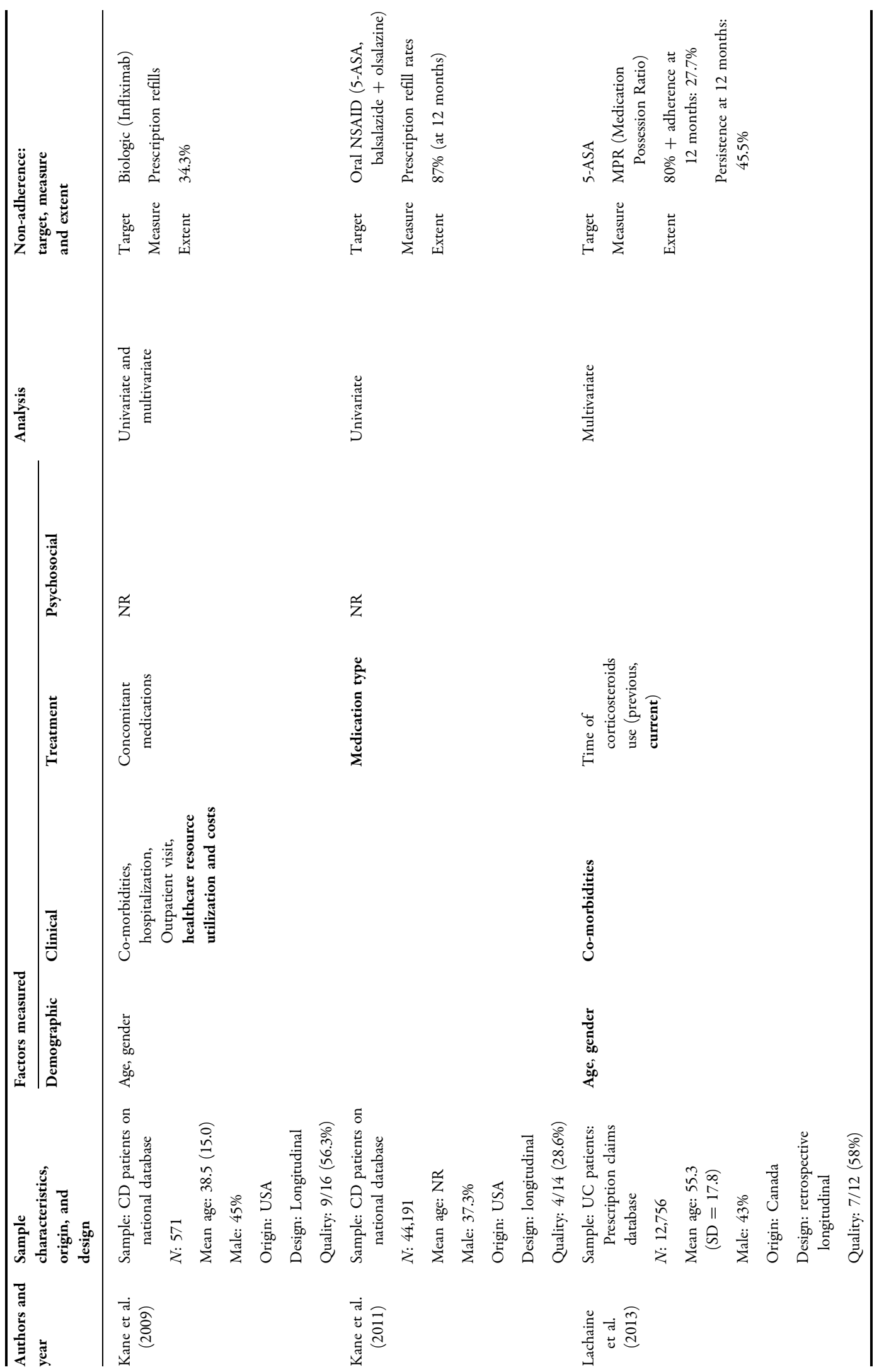




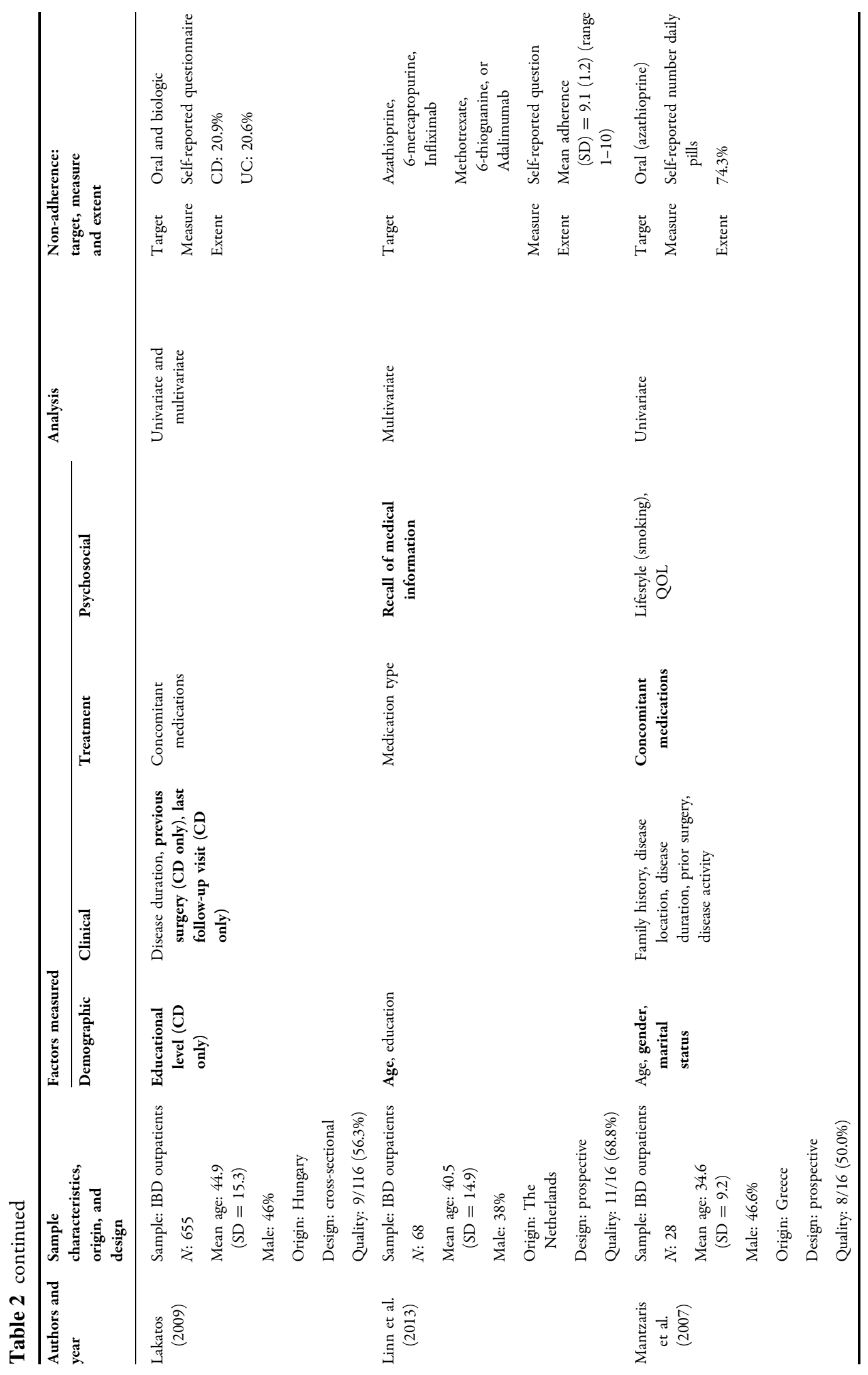




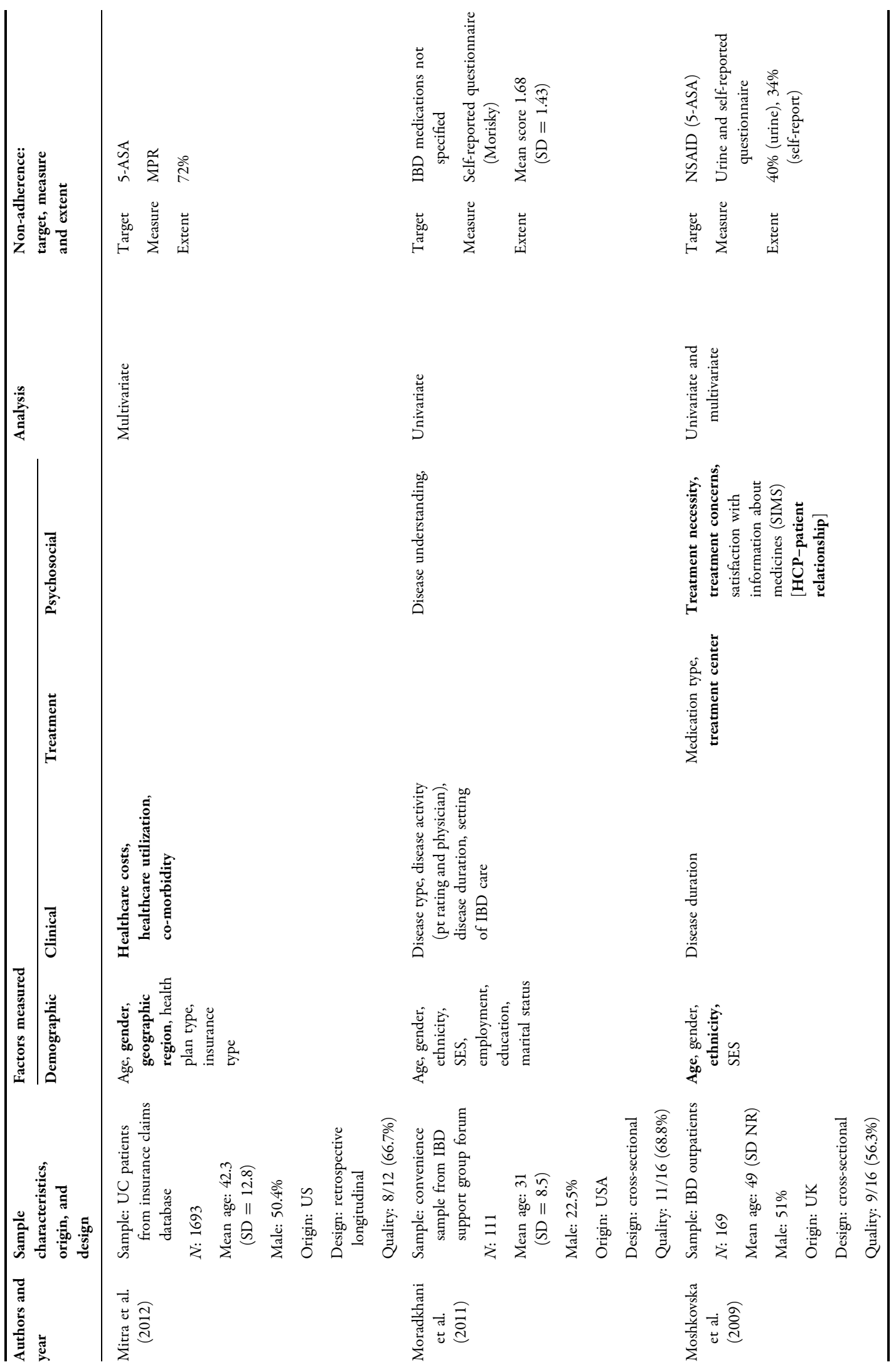




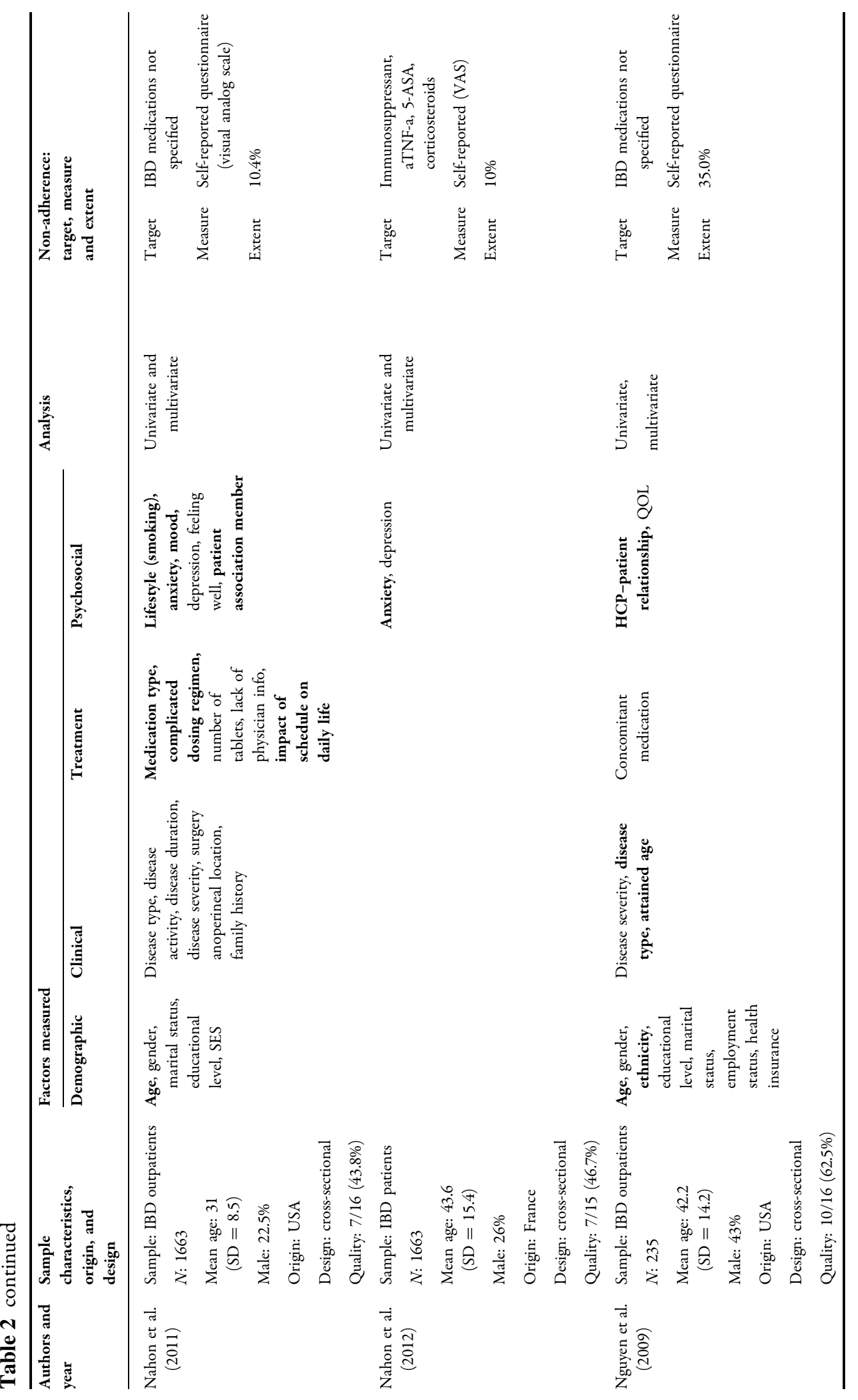




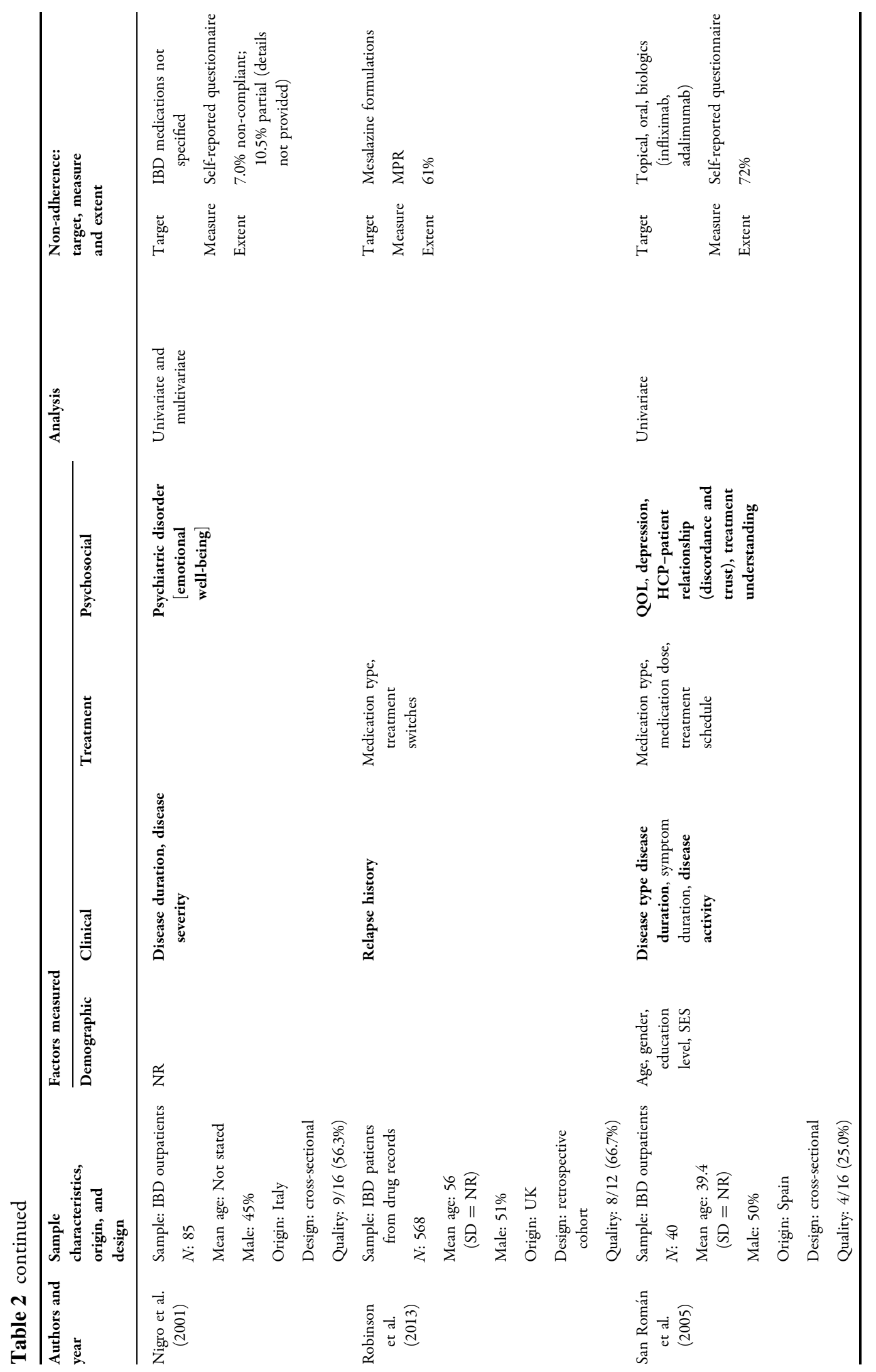




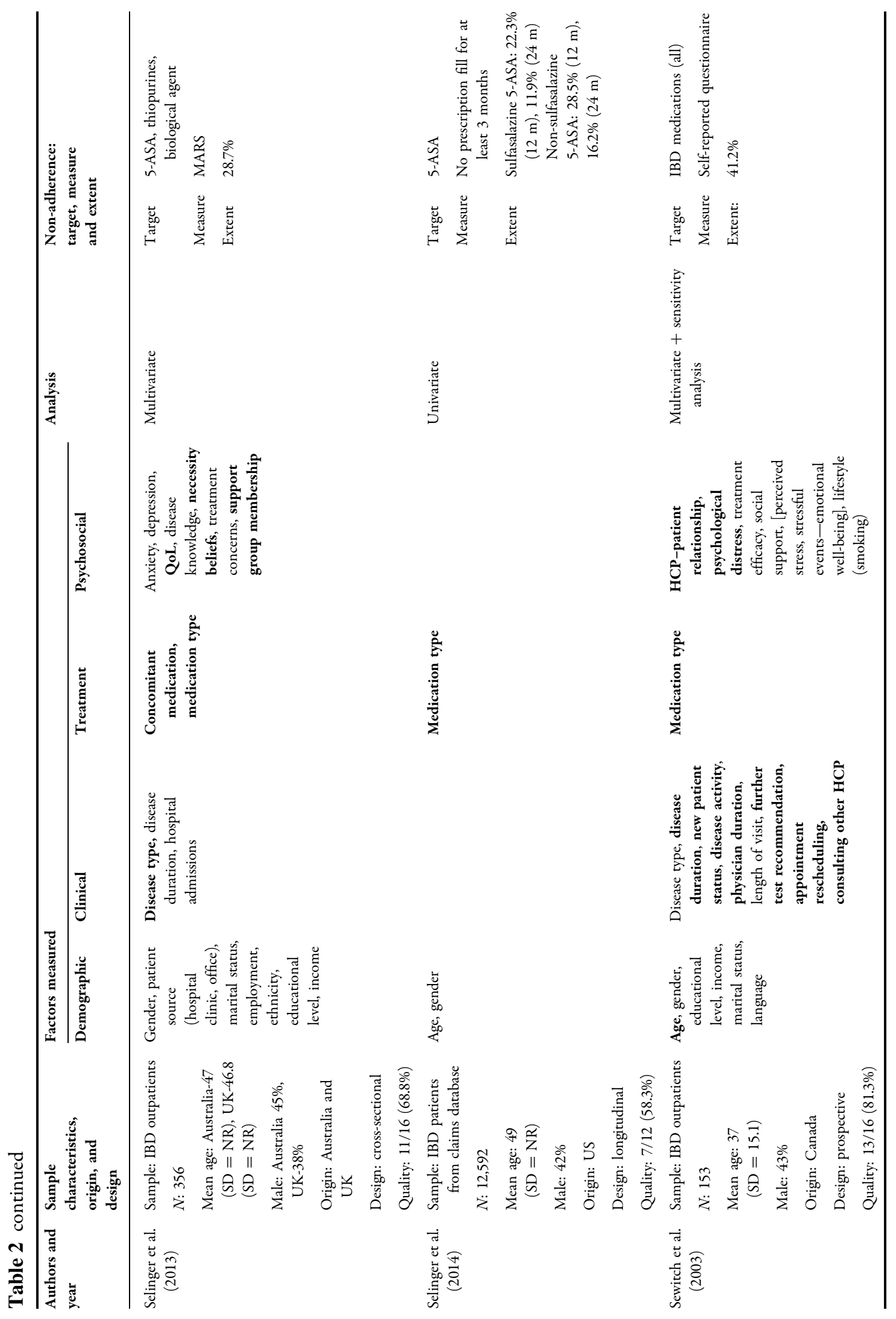




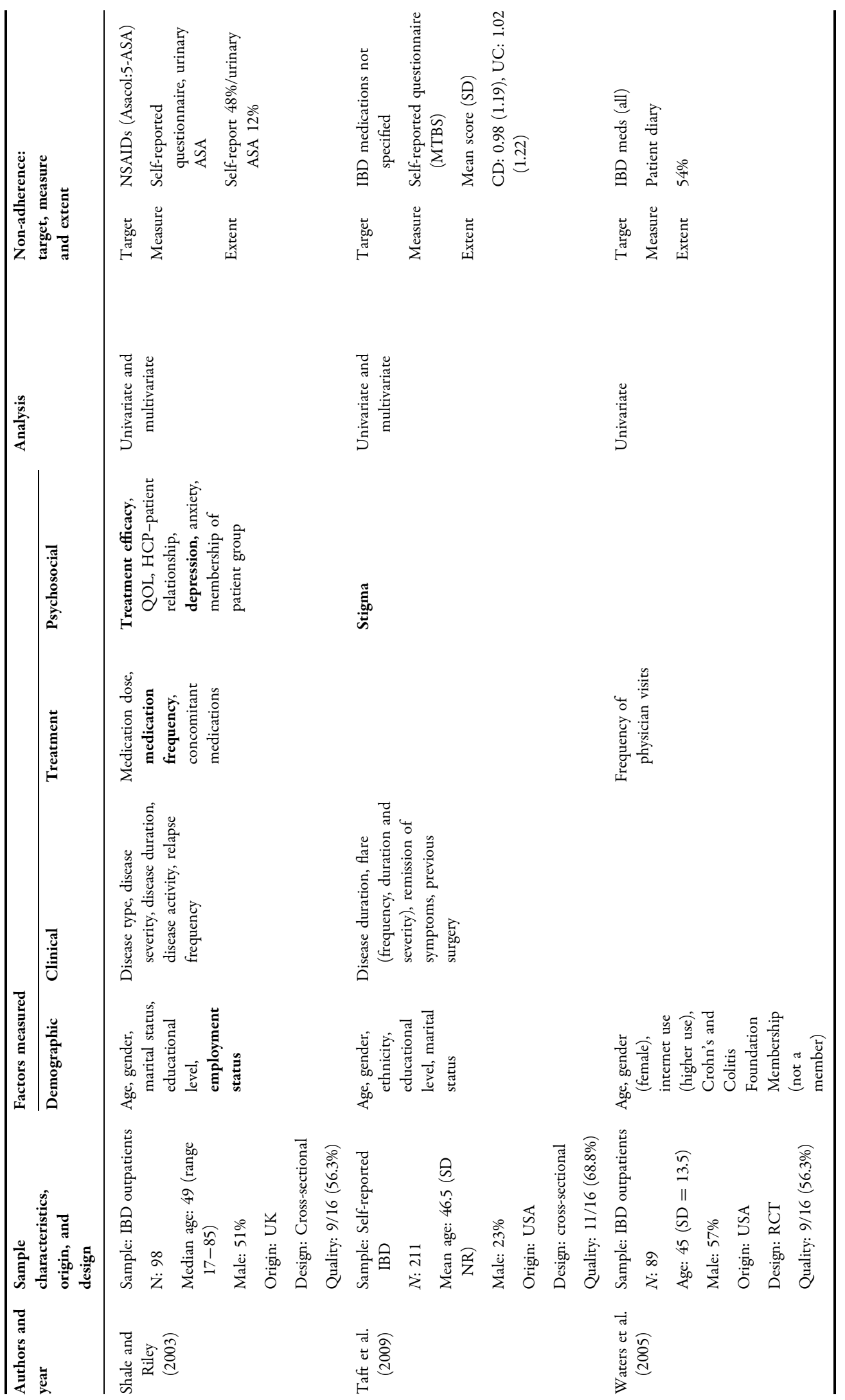




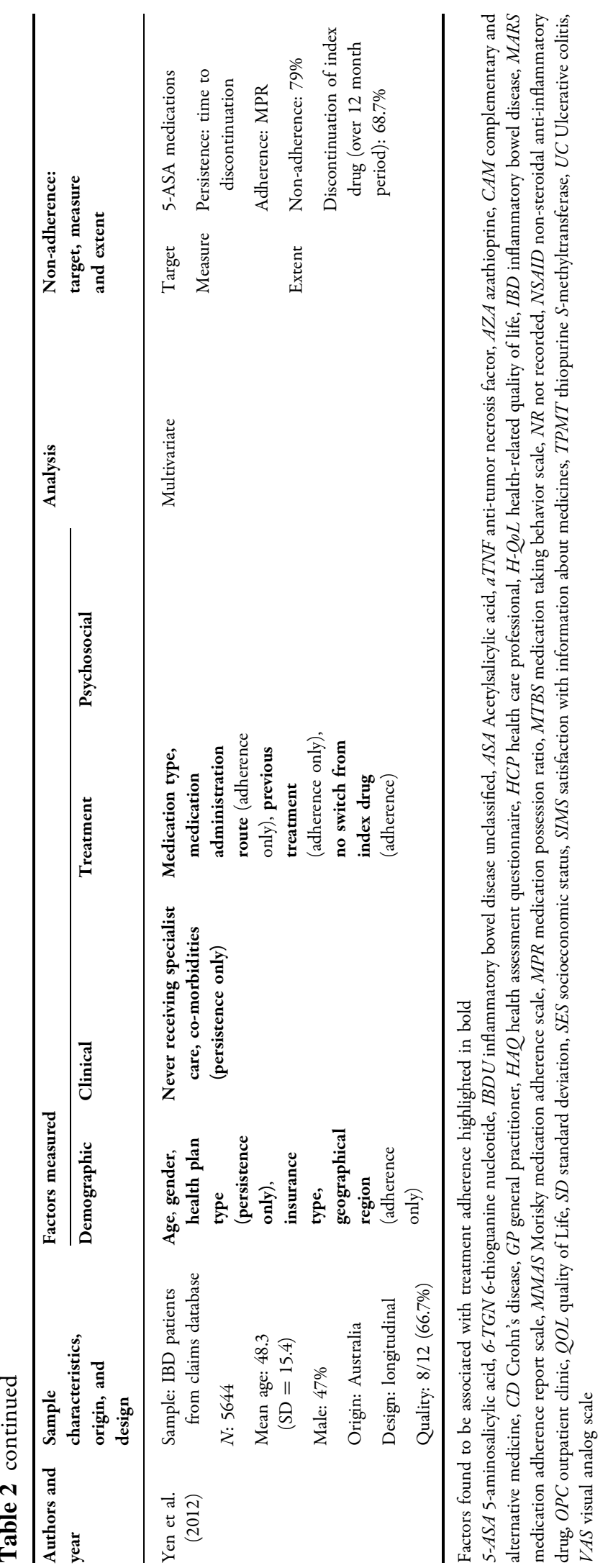




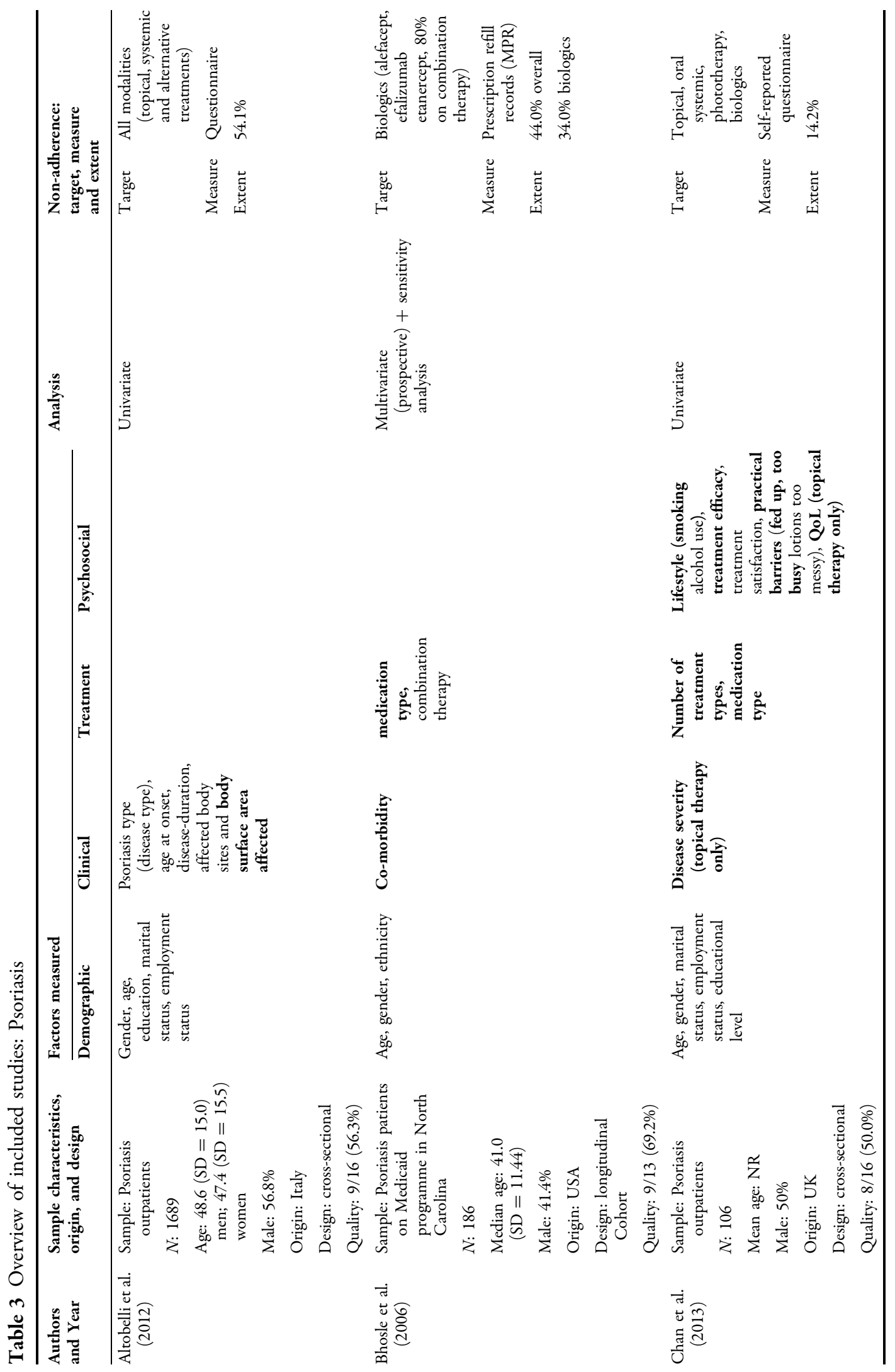




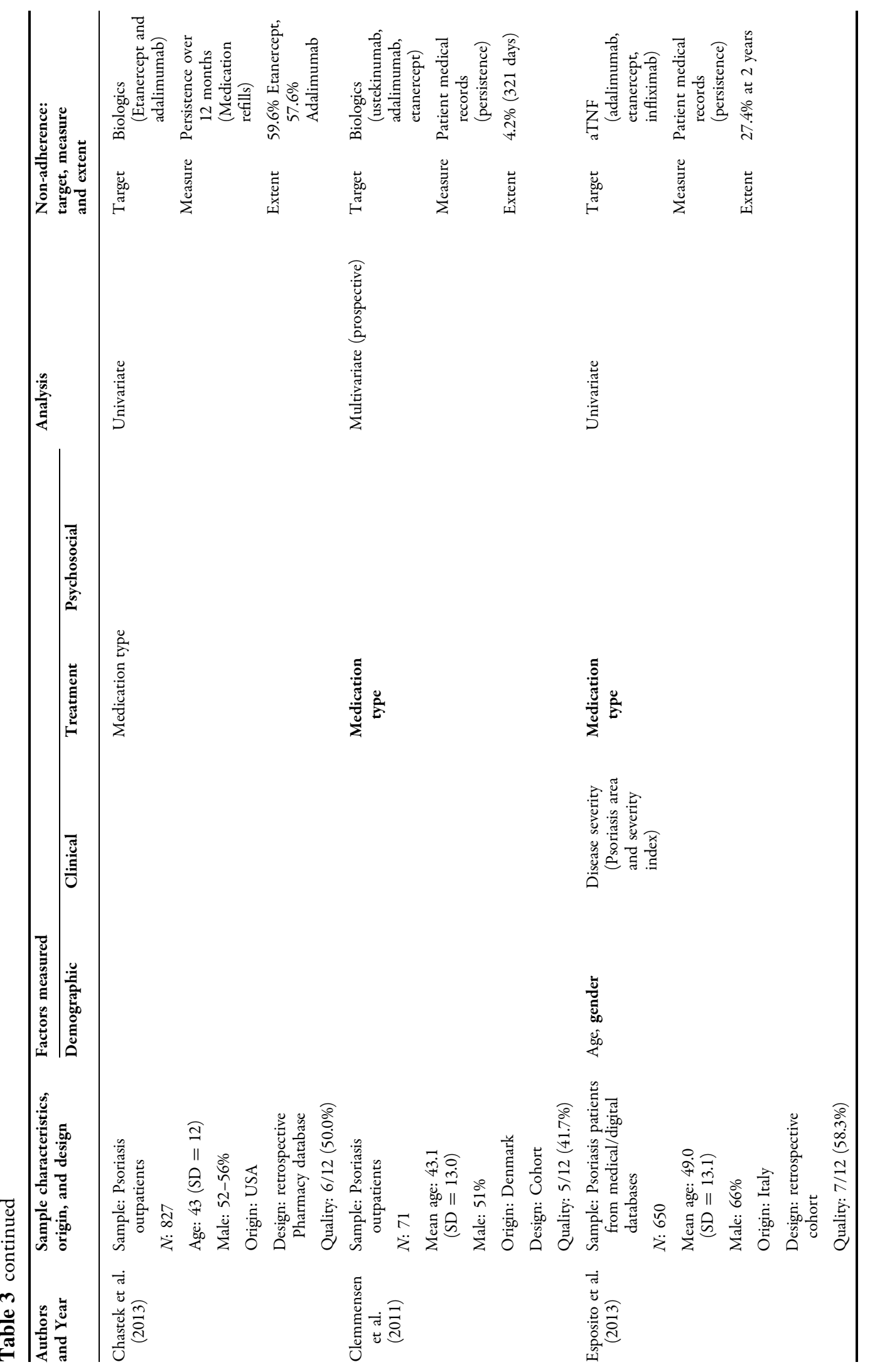




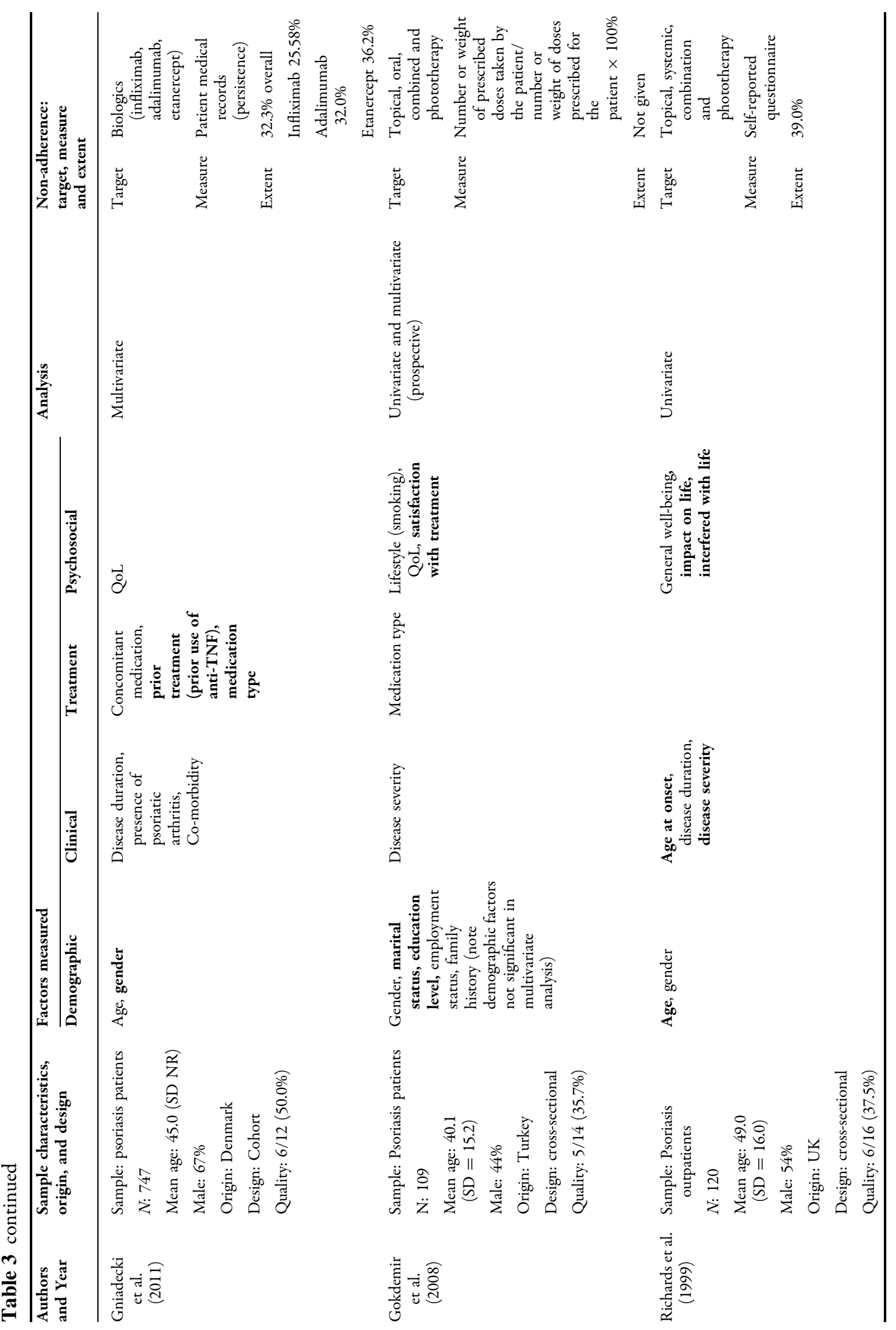




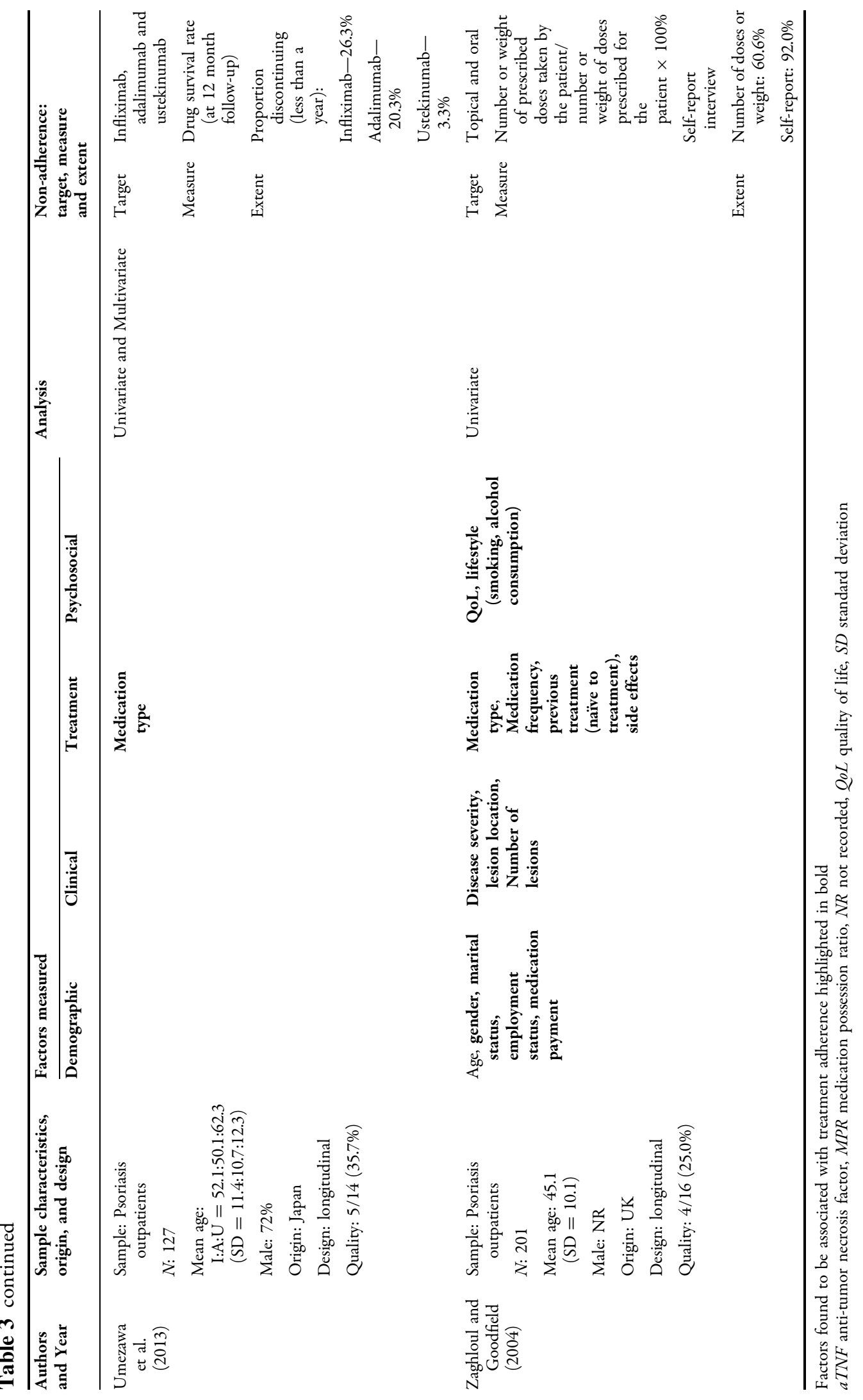




\section{Overview of Findings}

Adherence rates varied considerably in all clinical areas and ranged between $7 \%$ and $75 \%$ in $\mathrm{RC}, 4 \%$ and $72 \%$ in IBD, and $8 \%$ and $87 \%$ in PS. Evidence of an association of rates according to the adherence measure type (e.g., self-report, MEMs, biochemical, medication possession ratio) was not found. Factors assessed in relation to non-adherence were collated into four key categories: demographic; clinical; treatment; and psychosocial. All the factors explored across two or more chronic conditions, or in one condition and in a minimum of two studies with consistent results are presented in Table 4 . The table summarizes the frequency of studies examining these factors and proportion of studies to find a statistically significant association.

\section{Demographic Factors}

Age and gender were the most commonly examined factors $(79.5 \%$ and $80.8 \%)$ in relation to adherence across conditions. The majority of studies to examine them $(n=38$, $65.5 \%$ and $n=44,74.6 \%$, respectively) found no association with adherence and, where these were found, the findings were not consistent. The exception was for IBD where older age was found to be associated with greater likelihood of adherence in all studies to find an association $(n=11)$. However, an association was found in only a minority of the IBD studies; the majority (i.e., 18 out of 29) found age not to be associated with adherence. Marital status, education level, socioeconomic status, employment status, income, insurance type, geographical location and ethnicity were not consistently associated with non-adherence across diseases.

\section{Clinical Factors}

Clinical factors were the second most commonly examined (see Table 4). Disease duration and disease activity were the two clinical factors examined most frequently ( $n=37$ and $n=28)$. However, only a small proportion of these studies $(21.6 \%$ and $25 \%)$ found an association with adherence, and where associations were found, the relationship was not found to be consistent. In some cases, the relationship between disease duration and activity was positively associated with adherence, while in others there was a negative association. Disease severity and lesion location, although only examined in a minority of studies ( $n=10$ and $n=2$ ), reported the most consistent associations. In the PS studies, disease severity was the most commonly examined clinical factor in relation to adherence (45.5\%). An association with adherence was found in three of these studies $(60 \%)$, in which patients with lower disease severity were more likely to be non-adherent to their PS treatment than those with greater disease severity [84, 90, 92]. Only two of the five IBD studies (40\%) to examine this reported an association between disease severity and adherence and the direction of this association conflicted. None of the included RC studies examined disease severity.

Location of psoriatic lesions was examined in two of the PS studies (18\%). Non-adherence was found to be more likely among patients with facial lesions compared to those with lesions restricted to the rest of the body or with increasing number of lesion sites [92] and among those with greater body surface area of lesions [72]. Further details about these studies are available in the Tables 1, 2, 3 and 4 . 


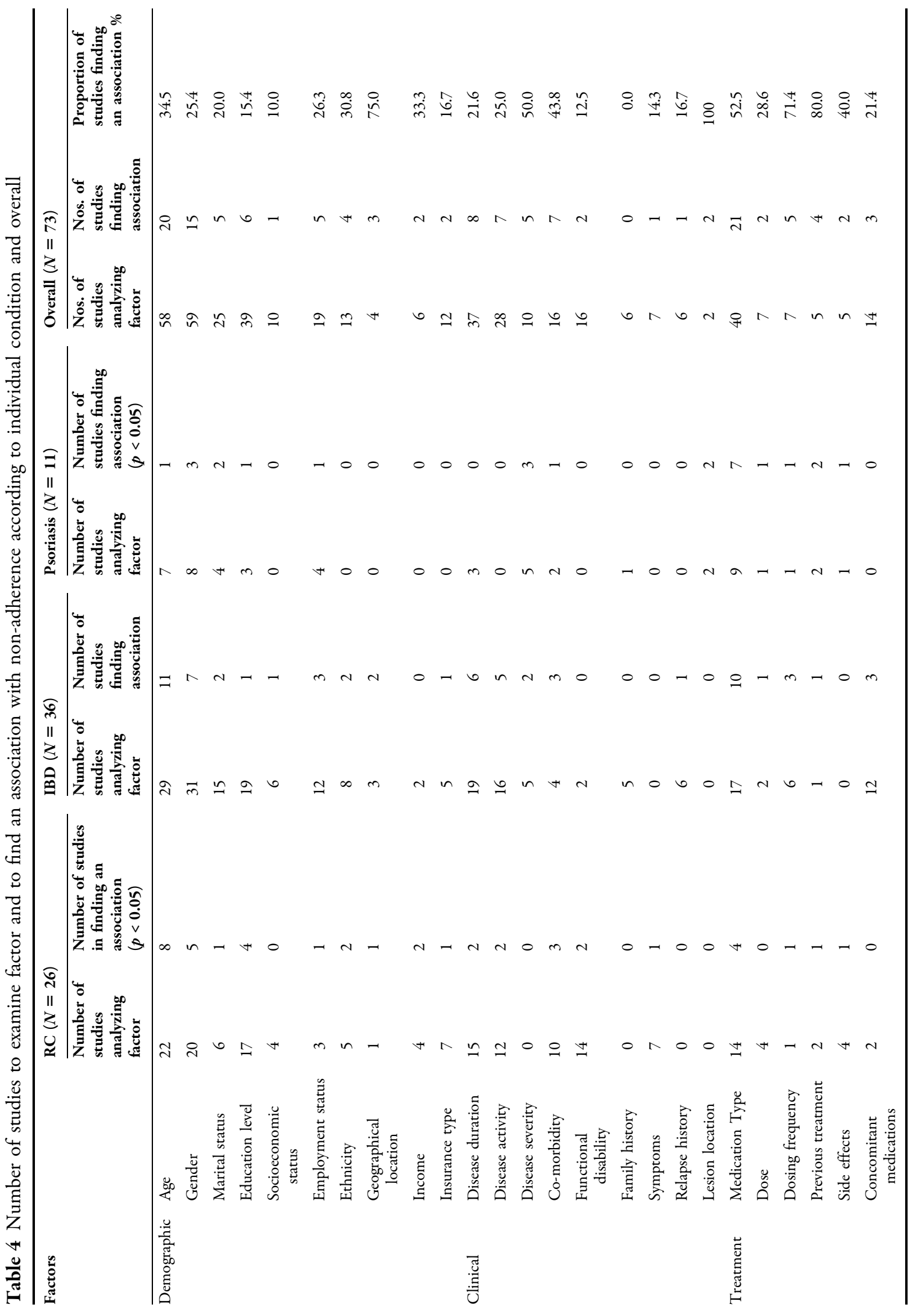




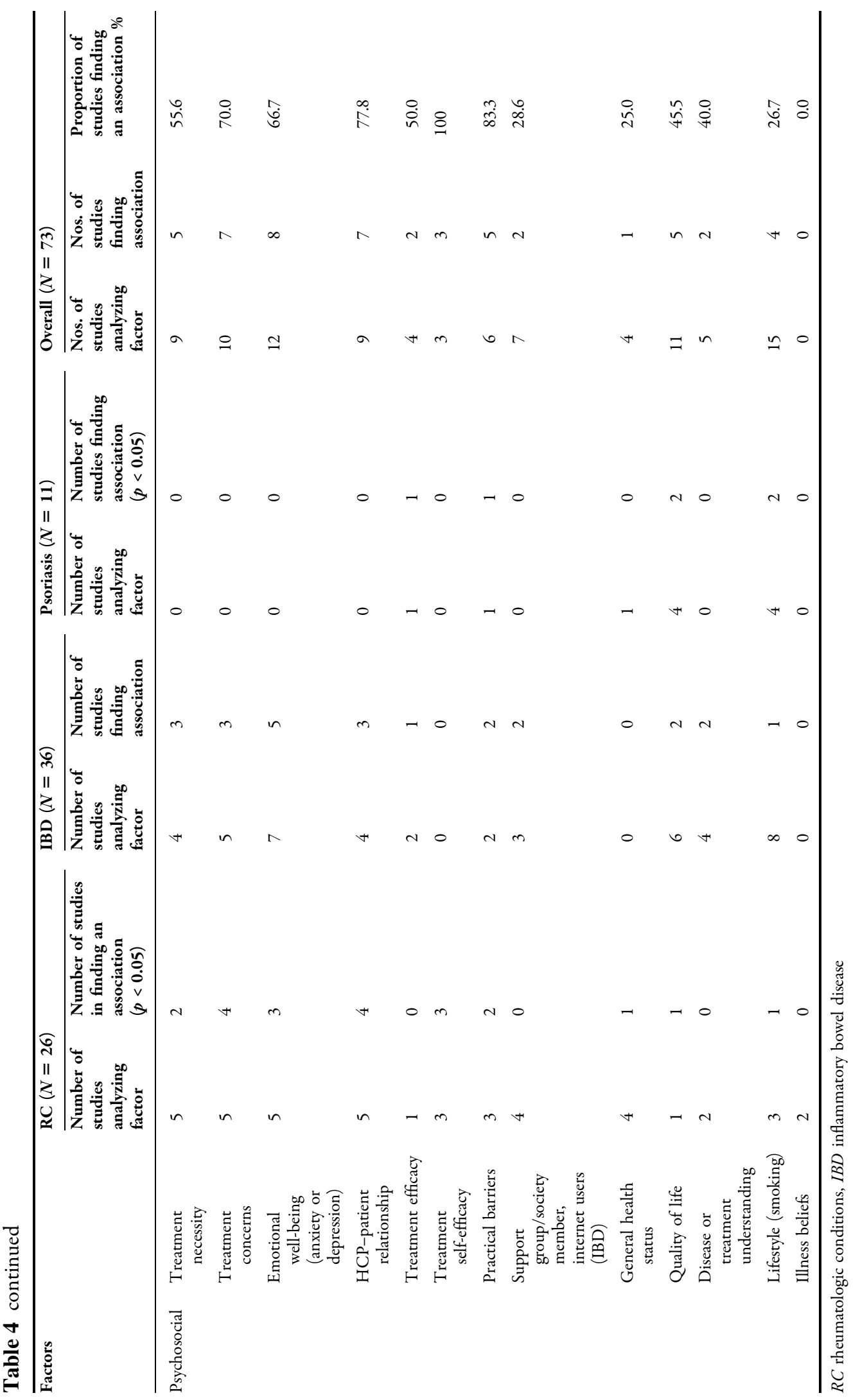




\section{Treatment Factors}

Medication type, dosing frequency, and previous treatment showed the most frequent association with adherence in the treatment category. Medication type was the most commonly explored treatment factor, which was assessed in 40 studies (54.8\%) with an association to non-adherence reported in over half of these studies (52.5\%).

In RC, non-steroidal anti-inflammatory drugs (NSAIDs) were found to be associated with lower adherence levels than disease-modifying medications [conventional synthetic disease-modifying anti-rheumatic drugs (csDMARDs)] in one study [28]. Another study found an association only for patients on steroids with these more likely to be adherent than those patients on NSAIDs or csDMARDs [40], although no association with corticosteroid use was observed in the other study examining this [36]. Among anti-tumor necrosis factor (TNF) treatments, significantly higher discontinuation rates and lower adherence levels were found for the biologic infliximab compared to the biologic etanercept and adalimumab [31, 38].

In IBD, greater adherence was associated with patients receiving anti-TNF (versus Prednisolone, Budesonide, exclusive enteral nutrition and 5-aminosalicylic acid (5-ASAs) [58], immunomodulator (versus 5-ASA) [46, $54,56]$, and steroid treatments compared to those who prescribed other medications (including 5-ASAs, immunosuppressants and antibiotics) [74]. In another study, non-adherence was reported to be more frequent in treatment with 5-ASAs compared to treatment with thiopurines and biological therapy [75]. Patients on oral treatment were more likely to be adherent compared to those on topical and enema treatments in another study [53].
Persistence rates were significantly higher for patients taking non-sulfasalazine 5-ASA compared to those taking sulfasalazine 5-ASA in one study [76], whereas in another study persistence was higher for those prescribed a multi-matrix system mesalamine 5-ASA compared to those prescribed balsalazide, mesalamine delayed release or sulfasalazine 5-ASAs [81]. In PS, six studies looked for associations according to biological DMARD, with higher persistence to adalimumab or etanercept compared to infliximab in one study [88], higher persistence to etanercept compared to both adalimumab and infliximab in another study [87] and higher persistence to ustekinumab compared with other anti-TNFs found in two studies $[86,91]$. The other two studies found no difference in levels of adherence between adalimumab and etanercept [85] or between alefacept, efalizumab or etanercept [83].

The number of doses taken daily was explored in seven studies across the diseases, of which the majority found an association $(71.4 \%, n=5)$. While the dosing frequencies examined varied between studies, associations were consistent, in that a greater likelihood of adherence to treatment was found with less frequent dosing.

Previous treatment was explored in five studies, four of which found an association with adherence (80\%). Three of these studies reported that previous exposure to the same drug or similar type of treatment increased the likelihood of non-adherence/early discontinuation [31, 88, 92]. This may be due to confounding factors such as lack of efficacy or acquired resistance to the drug class. The remaining study, reported that not having used rectal 5-ASA or immunosuppressive/biologic agents, was associated with the risk of non-persistence and non-adherence to 5-ASAs [81]. 


\section{Psychosocial Factors}

Thirteen psychosocial factors were examined in relation to adherence (see Table 4). Psychosocial factors were most commonly examined in the studies of IBD, followed by RC and were rarely examined in studies of PS. Treatment beliefs (i.e., necessity, concerns and efficacy), emotional well-being (depression and anxiety), HCP-patient relationship, treatment self-efficacy (i.e., confidence in one's ability to follow treatment) and practical barriers (e.g., frequent traveling, forgetfulness, etc.) were found to be associated with non-adherence in at least $50 \%$ of the studies to examine these. Non-adherence was found to be associated with doubts about treatment necessity in $55.6 \%$ of the studies to examine this $[29,40,57,68,75]$. Similarly, concerns about side effects and low perception of treatment efficacy were found to be associated with non-adherence in $70 \%$ and $50 \%$ of studies to examine this, respectively [29, $33,39,40,57,68,78,84]$. Four of the ten studies in RC and IBD to examine depression found a consistent association with non-adherence, with greater non-adherence reported amongst patients with depression or depressive symptoms. For anxiety, while over a third of the studies to examine this ( $n=3,37.5 \%)$ found an association with non-adherence, the direction of association was inconsistent. No studies assessed depression or anxiety in patients with PS. Practical barriers (e.g., frequent traveling, forgetfulness, etc.) were explored in six studies, and five of these found non-adherence to be more likely when practical barriers to taking treatment were perceived to be present. There was also some evidence that low levels of trust and satisfaction in the HCP-patient relationship may increase treatment non-adherence, with an association reported in $77.8 \%$ of the studies to examine this
$[32,37,40,43,71,74,77]$. This factor was not examined in any of the PS studies. Lower treatment self-efficacy was significantly and consistently associated with poorer medication adherence in all three studies of RC [23, 28, 39]. This factor was not examined in any of the IBD or PS studies.

\section{DISCUSSION}

This is the first review to systematically examine factors associated with non-adherence to treatment specifically for patients with selected IMIDs across three clinical areas. Demographic factors were the most commonly examined in relation to non-adherence followed by clinical and treatment factors. Psychosocial factors were examined in a minority of studies in RC and IBD and rarely examined in the PS studies. However, several consistent associations with adherence were observed for psychosocial factors that appear independent of the therapeutic area assessed.

While examined most commonly, none of the demographic or clinical factors were found to be consistently associated with non-adherence. Despite the general beliefs that some demographic factors are associated with non-adherence, this finding is in line with the other systematic literature reviews, where there is no consistent relationship between demographic characteristics and adherence in patients with chronic conditions [2-4]. Of the demographic factors, there was some evidence of an association between older age and adherence to IBD treatments; however, further studies are necessary to fully determine this.

With the clinical factors, there was some evidence that treatment non-adherence may be more likely among patients with PS with greater number/body surface area of lesions and among 
those with facial lesions in both studies to examine them. While the association of greater non-adherence with increased lesion coverage may appear counterintuitive, the visibility of psoriatic lesions to others well-being is put forward as a main stigmatizing factor from the patients' perspective which may have a significant impact on perceptions of body image and well-being [93], thus it is possible that the observed association is mediated by psychosocial factors such as anxiety or depression, the effects of which are discussed below. However, it is important to note the observed association is based on only two studies rated to be of medium to low quality.

Some evidence of an association was also found with the treatment factors including frequency of dosing and medication type. Due to wide heterogeneity in the medication types assessed, and scarce comparison studies among drug classes and between oral and injectable medications, it is not possible to draw conclusions as to which types of medication are associated with greater non-adherence. Consistent with some earlier studies [94, 95], less frequent dosing was associated with increased adherence, which may reflect the lower demand on memory and planning for the patient. However, it was not possible to assess whether there was a dosing frequency above which the likelihood of treatment non-adherence is increased, again due to wide heterogeneity in dosing frequencies assessed.

Psychosocial factors were only explored in a minority of studies. Despite heterogeneity in measures used, several consistent associations were observed. In particular, the current review found evidence that lower perceptions of treatment necessity $[29,40,57,68,75]$ and of treatment efficacy [78, 84], greater treatment concern [39, 78, 84] and higher HCP-patient discordance $[32,37,40,43,71]$ were associated with greater likelihood of non-adherence. Similar associations have been observed for necessity and concern beliefs about medication and the $\mathrm{HCP}$-patient relationship in previous reviews of adherence in IBD and RA specifically $[3,13,15,17]$, as well as in a systematic review across multiple conditions [96]. This suggests that addressing treatment concerns, increasing understanding of treatment necessity, and enhancing HCP-patient communication may be paramount to facilitate treatment adherence, irrespective of the type of IMID.

Evidence of an association of poorer emotional well-being, particularly depression, with non-adherence was found in the current review. Associations between anxiety and non-adherence on the other hand were less consistent, indicating that if an association exists, this may be weaker. These findings are consistent with those of a systematic review of studies of patients across a range of chronic conditions [97]. Both reviews suggest that depression but not anxiety may be a risk factor for treatment non-adherence in IMIDs, as well as chronic conditions more generally. This finding is of high importance, as depression is a potentially modifiable factor if diagnosed and treated appropriately, thus reducing the likelihood of poor adherence. It also raises an important question about the nature of the process in this effect. For example, depression might have effects on memory and planning ability, as well as on beliefs about treatment and efficacy $[97,98]$.

Treatment self-efficacy may also be an important factor for treatment adherence. Thus, patients with stronger beliefs in their ability to follow treatment were found to be more likely to adhere than those with comparatively weaker self-efficacy beliefs. 
Although, this was only examined in studies of $\mathrm{RC}$, previous systematic reviews have found treatment self-efficacy to be closely related to adherence in a number of different chronic conditions [96]. However, to enable firm conclusions to be drawn, further research is needed to investigate these factors among patients specifically with PS and IBD.

Evidence of an impact of practical barriers in treatment adherence was also found in the current review. The category of practical barriers is broad and can encompass many different types. The application of some topical creams in PS, for example, presents physical and possibly social barriers to administering treatment. Frequent traveling, busy lifestyles or forgetfulness may present time- and routine-related barriers. While these barriers on the surface may appear to be unintentional drivers of non-adherence, recent research has shown that patient perceptions of unintentional factors can be predicted by medication beliefs (intentional non-adherence factors [99]). This suggests that practical barriers may reflect in part reduced motivation to take treatment, and, as such, addressing treatment beliefs would also be necessary to overcome them. For this reason, practical barriers are incorporated into the broader category of psychosocial risk factors.

\section{Limitations and Recommendations for Future Research}

Limitations to this review and the quality of the available evidence should be taken into account when interpreting the findings. For example, it was not possible to draw conclusions regarding factors associated across a range of RCs, as the majority of studies eligible for inclusion were found for the condition RA. Similarly, it was not possible to draw conclusions as to whether factors associated with biologic systemic treatments were comparable to those of other classes of treatment, due to the lack of studies to examine this. Further, the majority of the assessed studies relied on patient-reported and thus subjective measures of adherence, which may not be an accurate reflection of true level of non-adherence. In addition, psychosocial factors were only explored in a minority of studies. As evidence for psychosocial factors was the most consistent, it is important for further research to focus on understanding the nature and strength of the relationship of these factors with treatment adherence. In particular, there is a strong need for prospective longitudinal studies to determine whether the factors identified in the current review predict treatment non-adherence or are related in another way. Similarly, there is also a need for intervention studies in which these factors are modified to see whether this results in improved adherence.

The high level of heterogeneity in both the measures and analysis approaches applied across studies limits the conclusions that can be drawn from the synthesis of the data. Although the type of measure did not correlate with the overall level of adherence found, it was not possible to determine whether the pattern of associations varied according to the adherence measure used. There was wide variation in the quality of the studies, which may have influenced the pattern of findings. Studies investigating adherence across a range of IMID conditions using the same measures and analysis approach are urgently needed to enable identification of common and consistent predictors. Efforts to address such limitations are currently underway in the ALIGN study; a multi-country, cross-section AL study to determine patient specific and General beliefs towards medication and their treatment 
adherence to selected systemic therapies in chronic inflammatory diseases (IMID) (UKCRN ID: 12782). It is anticipated that the results of this study will build on the findings of this review and further advance our understanding of the role of factors particularly those of patient beliefs about treatment for adherence to systemic therapies.

\section{Implications for Clinical Practice}

Although the heterogeneity of the reviewed studies restricts our ability to draw firm conclusions, fairly minimal evidence was found for an impact of demographic, clinical and treatment factors on treatment non-adherence, but more consistent evidence was reported for psychosocial factors. The findings of the current review indicate that greater treatment concern, lower treatment self-efficacy and necessity, presence of depression or practical barriers, and a sub-optimal HCP-patient relationship may have a negative impact on treatment adherence, and these could be considered modifiable risk factors. At present, assessing adherence is not always high priority within clinical practice, due in part to a combination of time constraints and lack of awareness regarding the extent and nature of the problem. Even when adherence is measured, the rates can be of limited value without the understanding of potential risk factors, and most importantly, interventions to address and modify these psychosocial factors. Increasing clinician awareness of the adherence problem, however, does not always result in changes to patient beliefs and behavior [100]. Twofold intervention, that focuses on increasing clinician awareness about the range of factors, particularly psychosocial that may impact on treatment non-adherence as well as tools to help tackle these issues, are thus likely to be most effective. For example, the development of tools to help clinicians elicit and address patient beliefs in routine consultations is recommended.

\section{CONCLUSIONS}

Through a systematic analysis of the evidence across the clinical areas of RC, IBD and PS, this review has identified common patterns to both focus research efforts and to support the development of tools or interventions in routine care to help patients follow their prescribed treatment regimen. To date, the main focus of research in the areas of RC, IBD and PS has been on the association of demographic factors, and clinical or treatment factors. The findings of the current review, however, suggest that these factors are not consistent or key determinants of adherence. It appears that psychosocial factors are more consistently associated with adherence. As such, interventions designed to modify these factors through addressing treatment beliefs, providing practical advice on taking treatment, and improving communication between HCPs and patients may prove to be the most effective.

\section{ACKNOWLEDGMENTS}

EV and JW designed the study and SB the study materials. $\mathrm{SB}, \mathrm{AF}, \mathrm{DB}, \mathrm{AB}$ and $\mathrm{EV}$ collected the data and conducted an analysis of the individual reviews. $\mathrm{EV}, \mathrm{JW}, \mathrm{LPB}, \mathrm{UM}, \mathrm{AO}$, and AL interpreted the data. All authors contributed to the revised manuscript and provided their approval of the final draft. Editorial assistance in the preparation of this manuscript was provided by Dr. Sumaira Malik of Atlantis Healthcare. Financial support for this study, article processing charges and open access fee was funded by AbbVie. AbbVie reviewed and 
approved the final publication. All named authors meet the ICMJE criteria for authorship for this manuscript, take responsibility for the integrity of the work as a whole, and have given final approval to the version to be published.

Disclosures. Dr Eleni Vangeli received funding from pharmaceutical companies for the research and development of patient support programs as a former employee of Atlantis Healthcare. Dr. Savita Bakhshi is a former employee of Atlantis Healthcare, and received funding from Abbott/AbbVie to conduct the current review, and from other pharmaceutical companies for the research and development of patient adherence and support programs. Dr. Anna Baker as a former employee of Atlantis Healthcare received funding from a number of pharmaceutical companies for the research and development of patient adherence and support programs, including funding from Abbott/AbbVie to conduct the current review. Dr. Abigail Fisher received funding from AbbVie to conduct the systematic review. Delaney Bucknor has received payment from Atlantis Healthcare as a freelance researcher to undertake a component of the systematic review submitted for publication. Dr. Andrew Östör has received support from (including attendance at conferences), undertakes clinical trials and acts as a consultant to Roche, Chugai, MSD, Abbvie, Pfizer, Napp \& BMS. Prof. Mrowietz has been an advisor and/ or received speakers honoraria and/or received grants and/or participated in clinical trials of the following companies: Abbott/AbbVie, Almirall-Hermal, Amgen, BASF, Biogen Idec, Celgene, Centocor, Eli Lilly, Forward Pharma, Galderma, Janssen, Leo Pharma, Medac, MSD, Miltenyi Biotech, Novartis, Pfizer, Teva, VBL, Xenoport. Prof. Peyrin-Biroulet reports having received consulting and/or lecture fees from AbbVie, Actelion, Bristol-Myers Squibb, Celgène, Ferring Pharmaceuticals, Janssen, Merck, Mitsubishi, Shire Pharmaceuticals, Therakos, Norgine, Pharmacosmos, Pilège, Therakos, Tillotts Pharma AG (acquired by Zeria Pharmaceutical Co., Ltd) and Vifor. Ana $P$ Lacerda Ana Lacerda is an employee of AbbVie Inc, and may hold AbbVie stock or options. Prof. Weinman is also employed by Atlantis Healthcare, which provides patient adherence and support programs, which are funded by a number of pharmaceutical companies.

Compliance with Ethical Guidelines. This article is based on previously conducted studies and does not involve any new studies of human or animal subjects performed by any of the authors.

Open Access. This article is distributed under the terms of the Creative Commons Attribution-NonCommercial 4.0 International License (http://creativecommons.org/licenses/ by-nc/4.0/), which permits any noncommercial use, distribution, and reproduction in any medium, provided you give appropriate credit to the original author(s) and the source, provide a link to the Creative Commons license, and indicate if changes were made.

\section{REFERENCES}

1. Kuek A, Hazleman BL, Östör AJ. Immune-mediated inflammatory diseases (IMIDs) and biologic therapy: a medical revolution. Postgraduate Med J. 2007;83(978): 251-60.

2. van den Bemt BJ, Zwikker HE, van den Ende $\mathrm{CH}$. Medication adherence in patients with rheumatoid arthritis: a critical appraisal of the existing literature. Expert Rev Clin Immunol. 2012;8(4):337-51. 
3. Jackson C, et al. Factors associated with non-adherence to oral medication for inflammatory bowel disease: a systematic review. Am J Gastroenterol. 2009;105(3):525-39.

4. Thorneloe R, et al. Adherence to medication in patients with psoriasis: a systematic literature review. Br J Dermatol. 2013;168(1):20-31.

5. Haynes $\mathrm{RB}$, et al. Interventions for enhancing medication adherence. Cochrane Database Syst Rev. 2008:(2):CD000011.

6. Krueger KP, Berger BA, Felkey B. Medication adherence and persistence: a comprehensive review. Adv Ther. 2005;22(4):313-56.

7. World Health Organisation. Adherence to long-term therapies: evidence for action. Geneva: WHO; 2003.

8. Augustin M, et al. Adherence in the treatment of psoriasis: a systematic review. Dermatology. 2011;222(4):363-74.

9. Blum MA, Koo D, Doshi JA. Measurement and rates of persistence with and adherence to biologics for rheumatoid arthritis: a systematic review. Clin Therapeut. 2011;33(7):901-13.

10. Devaux S, et al. Adherence to topical treatment in psoriasis: a systematic literature review. J Eur Acad Dermatol Venereol. 2012;26(s3):61-7.

11. Feinberg J. The effect of patient-practitioner interaction on compliance: a review of the literature and application in rheumatoid arthritis. Patient Educ Couns. 1988;11(3):171-87.

12. Kane S. Systematic review: adherence issues in the treatment of ulcerative colitis. Aliment pharmacol Therapeut. 2006;23(5):577-85.

13. Hawthorne A, Rubin G, Ghosh S. Review article: medication non-adherence in ulcerative colitis-strategies to improve adherence with mesalazine and other maintenance therapies. Aliment Pharmacol Therapeut. 2008;27(12):1157-66.

14. Koncz T, et al. Adherence to biologic DMARD therapies in rheumatoid arthritis. Expert Opin Biol Ther. 2010;10(9):1367-78.

15. Salt E, Frazier SK. Adherence to disease-modifying antirheumatic drugs in patients with rheumatoid arthritis: a narrative review of the literature. Orthop Nurs. 2010;29(4):260-75.
16. Fiddler $\mathrm{HH}$, Singendonk MMJ, van der Have $M$, Oldenburg B, van Oijen MGH. Low rates of adherence for tumor necrosis factor- $\alpha$ inhibitors in Crohn's disease and rheumatoid arthritis: results of a systematic review. World J Gastroenterol WJG. 2013;19(27):7.

17. Pasma A, et al. Factors associated with adherence to pharmaceutical treatment for rheumatoid arthritis patients: a systematic review. In: Seminars in arthritis and rheumatism. 2013. Elsevier.

18. National Institute for Clinical Excellence. Methods for the development of NICE public health guidance. NICE: London; 2006

19. Moher D, Liberati A, Tetzlaff J, Altman DG. Preferred reporting items for systematic reviews and meta-analyses: the PRISMA statement. Ann Intern Med. 2009;151(4):264-9.

20. Arturi P, et al. Adherence to treatment in patients with ankylosing spondylitis. Clin Rheumatol. 2013;32(7):1007-15.

21. Beck N, et al. Patients with rheumatoid arthritis at high risk for noncompliance with salicylate treatment regimens. J Rheumatol. 1988;15(7):1081.

22. Borah BJ, et al. Trends in RA patients' adherence to subcutaneous anti-TNF therapies and costs. Curr Med Res Opin. 2009;25(6):1365-77.

23. Brus $\mathrm{H}$, et al. Determinants of compliance with medication in patients with rheumatoid arthritis: the importance of self-efficacy expectations. Patient Educ Counseling. 1999;36(1):57-64.

24. Caplan L et al. Health literacy is strongly associated with functional status among rheumatoid arthritis patients: a cross-sectional study. Arthritis Care Res. 2014;66(4):508-14.

25. Chastek B, et al. Etanercept and adalimumab treatment patterns in psoriatic arthritis patients enrolled in a commercial health plan. Adv Ther. 2012;29(8):691-7.

26. Cho S-K, et al. Impact of comorbidities on TNF inhibitor persistence in rheumatoid arthritis patients: an analysis of Korean National Health Insurance claims data. Rheumatol Int. 2012;32(12):3851-6.

27. Curkendall $\mathrm{S}$, et al. Compliance with biologic therapies for rheumatoid arthritis: do patient out-of-pocket payments matter? Arthritis Care Res. 2008;59(10):1519-26. 
28. de Klerk E, et al. Patient compliance in rheumatoid arthritis, polymyalgia rheumatica, and gout. J Rheumatol. 2003;30(1):44-54.

29. de Thurah A, et al. Compliance with methotrexate treatment in patients with rheumatoid arthritis: influence of patients' beliefs about the medicine. A prospective cohort study. Rheumatol Int. 2010;30(11):1441-8.

30. Garcia-Gonzalez A, et al. Treatment adherence in patients with rheumatoid arthritis and systemic lupus erythematosus. Clin Rheumatol. 2008;27(7):883-9.

31. Martínez-Santana V, et al. Comparison of drug survival rates for tumor necrosis factor antagonists in rheumatoid arthritis. Patient Preference Adherence. 2013;7:719.

32. Müller R, et al. Compliance with treatment of rheumatoid arthritis. Rheumatol Int. 2012;32(10):3131-5.

33. Neame R, Hammond A. Beliefs about medications: a questionnaire survey of people with rheumatoid arthritis. Rheumatology. 2005;44(6):762-7.

34. Park D, et al. Medication adherence in rheumatoid arthritis patients: older is wiser. J Am Geriatrics Soc. 1999;47:172-83.

35. Pascual-Ramos V, Contreras-Yáñez I. Motivations for inadequate persistence with disease modifying anti-rheumatic drugs in early rheumatoid arthritis: the patient's perspective. BMC Musculoskeletal Disord. 2013;14(1):336.

36. Pascual-Ramos V, et al. Medication persistence over 2 years of follow-up in a cohort of early rheumatoid arthritis patients: associated factors and relationship with disease activity and with disability. Arthritis Res Ther. 2009;11(1):R26.

37. Quinlan P, et al. The relationship among health literacy, health knowledge, and adherence to treatment in patients with rheumatoid arthritis. HSS Journal®. 2013;9(1):42-9.

38. Saad AA, et al. Persistence with anti-tumour necrosis factor therapies in patients with psoriatic arthritis: observational study from the British Society of Rheumatology Biologics Register. Arthritis Res Ther. 2009;11(2):R52.

39. Spruill TM, et al. Association of medication beliefs and self-efficacy with adherence in urban Hispanic and African-American rheumatoid arthritis patients. Ann Rheumatic Dis. 2014;73(1):317-8.
40. Treharne G, Lyons A, Kitas G. Medication adherence in rheumatoid arthritis: effects of psychosocial factors. Psychol Health Med. 2004;9(3):337-49.

41. Tuncay R, et al. Factors affecting drug treatment compliance in patients with rheumatoid arthritis. Rheumatol Int. 2007;27(8):743-6.

42. van den Bemt BJ, et al. Adherence rates and associations with nonadherence in patients with rheumatoid arthritis using disease modifying antirheumatic drugs. J Rheumatol. 2009;36(10):2164-70.

43. Viller $\mathrm{F}$, et al. Compliance to drug treatment of patients with rheumatoid arthritis: a 3 year longitudinal study. J Rheumatol. 1999;26(10):2114-22.

44. Waimann CA, et al. Electronic monitoring of oral therapies in ethnically diverse and economically disadvantaged patients with rheumatoid arthritis: consequences of low adherence. Arthritis Rheumat. 2013;65(6):1421-9.

45. Wong $M$, Mulherin $D$. The influence of medication beliefs and other psychosocial factors on early discontinuation of disease-modifying anti-rheumatic drugs. Musculoskeletal Care. 2007;5(3):148-59.

46. Bermejo F, et al. Factors that modify therapy adherence in patients with inflammatory bowel disease. J Crohn's Colitis. 2010;4(4):422-6.

47. Bernal I, et al. Medication-taking behavior in a cohort of patients with inflammatory bowel disease. Digestive Dis Sci. 2006;51(12):2165-9.

48. Billioud $\mathrm{V}$, et al. Adherence to adalimumab therapy in Crohn's disease: a French multicenter experience. Inflamm Bowel Dis. 2011;17(1): 152-9.

49. Bokemeyer B, et al. Adherence to thiopurine treatment in out-patients with Crohn's disease. Aliment Pharmacol Therapeut. 2007;26(2):217-25.

50. Carter CT, Waters HC, Smith DB. Effect of a continuous measure of adherence with infliximab maintenance treatment on inpatient outcomes in Crohn's disease. Patient Preference Adherence. 2012;6:417.

51. Červený $\mathrm{P}$, et al. Nonadherence in inflammatory bowel disease: results of factor analysis. Inflammatory Bowel Dis. 2007;13(10):1244-9. 
52. Cerveny $\mathrm{P}$, et al. Non-adherence to treatment in inflammatory bowel disease in Czech Republic. J Crohn's Colitis. 2007;1(2):77-81.

53. D'Inca R, et al. Risk factors for non-adherence to medication in inflammatory bowel disease patients. Aliment Pharmacol Therapeut. 2008;27(2):166-72.

54. Ediger JP, et al. Predictors of medication adherence in inflammatory bowel disease. Am J Gastroenterol. 2007;102(7):1417-26.

55. Goodhand $\mathrm{J}$, et al. Factors associated with thiopurine non-adherence in patients with inflammatory bowel disease. Aliment Pharmacol Therapeut. 2013;38(9):1097-108.

56. Horváth $\mathrm{G}$, et al. Is there any association between impaired health-related quality of life and non-adherence to medical therapy in inflammatory bowel disease? Scand J Gastroenterol. 2012;47(11):1298-303.

57. Horne R, et al. Patients' attitudes to medicines and adherence to maintenance treatment in inflammatory bowel disease. Inflamm Bowel Dis. 2009;15(6):837-44.

58. Kamperidis N, et al. Factors associated with nonadherence to thiopurines in adolescent and adult patients with inflammatory bowel disease. J Pediatr Gastroenterol Nutr. 2012;54(5):685-9.

59. Kane SV, et al. Twelve-month persistency with oral 5-aminosalicylic acid therapy for ulcerative colitis: results from a large pharmacy prescriptions database. Dig Dis Sci. 2011;56(12):3463-70.

60. Kane SV, Chao J, Mulani PM. Adherence to infliximab maintenance therapy and health care utilization and costs by Crohn's disease patients. Adv Therapy. 2009;26(10):936-46.

61. Kane SV, et al. Prevalence of nonadherence with maintenance mesalamine in quiescent ulcerative colitis. Am J Gastroenterol. 2001;96(10):2929-33.

62. Lachaine $\mathrm{J}$, et al. Medication adherence and persistence in the treatment of Canadian ulcerative colitis patients: analyses with the RAMQ database. BMC Gastroenterol. 2013;13(1):23.

63. Lakatos PL. Prevalence, predictors, and clinical consequences of medical adherence in IBD: how to improve it? World J Gastroenterol WJG. 2009;15(34):4234.

64. Linn AJ, et al. May you never forget what is worth remembering: the relation between recall of medical information and medication adherence in patients with inflammatory bowel disease. J Crohn's Colitis. 2013;7(11):e543-50.

65. Mantzaris GJ, et al. How adherent to treatment with azathioprine are patients with Crohn's disease in long-term remission? Inflam Bowel Dis. 2007; 13(4):446-50.

66. Mitra D, et al. Association between oral 5-ASA adherence and health care utilization and costs among patients with active ulcerative colitis. BMC Gastroenterol. 2012;12(1):132.

67. Moradkhani A, et al. Disease-specific knowledge, coping, and adherence in patients with inflammatory bowel disease. Digest Dis Sci. 2011;56(10):2972-7.

68. Moshkovska $\mathrm{T}$, et al. An investigation of medication adherence to 5-aminosalicylic acid therapy in patients with ulcerative colitis, using self-report and urinary drug excretion measurements. Aliment Pharmacol Ther. 2009;30(11-12):1118-27.

69. Nahon S, et al. Socioeconomic and psychological factors associated with nonadherence to treatment in inflammatory bowel disease patients: results of the ISSEO survey. Inflamm Bowel Dis. 2011;17(6):1270-6.

70. Nahon S, et al. Risk factors of anxiety and depression in inflammatory bowel disease. Inflamm Bowel Dis. 2012;18(11):2086-91.

71. Nguyen GC, et al. Patient trust-in-physician and race are predictors of adherence to medical management in inflammatory bowel disease. Inflamm Bowel Dis. 2009;15(8):1233-9.

72. Nigro G, et al. Psychiatric predictors of noncompliance in inflammatory bowel disease: psychiatry and compliance. J Clin Gastroenterol. 2001;32(1):66-8.

73. Robinson A, et al. Maintaining stable symptom control in inflammatory bowel disease: a retrospective analysis of adherence, medication switches and the risk of relapse. Aliment Pharmacol Therapeut. 2013;38(5):531-8.

74. Román San. Adherence to treatment in inflammatory bowel disease. Rev Esp Enferm Dig. 2005;97(4):249-57.

75. Selinger $\mathrm{CP}$, et al. modifiable factors associated with nonadherence to maintenance medication for inflammatory bowel disease. Inflamm Bowel Dis. 2013;19(10):2199-206.

76. Selinger CP, Kemp A, Leong RW. Persistence to oral 5-aminosalicylate therapy for inflammatory 
bowel disease in Australia. Expert Rev Gastroenterol Hepatol. 2014;1:6.

77. Sewitch MJ, et al. Patient nonadherence to medication in inflammatory bowel disease. Am J Gastroenterol. 2003;98(7):1535-44.

78. Shale M, Riley S. Studies of compliance with delayed-release mesalazine therapy in patients with inflammatory bowel disease. Aliment Pharmacol Therapeut. 2003;18(2):191-8.

79. Taft $\mathrm{TH}$, et al. Impact of perceived stigma on inflammatory bowel disease patient outcomes. Inflamm Bowel Dis. 2009;15(8):1224-32.

80. Waters BM, Jensen L, Fedorak RN. Effects of formal education for patients with inflammatory bowel disease: a randomized controlled trial. J Can Gastroenterol. 2005;19(4):235-44.

81. Yen L, et al. Medication use patterns and predictors of nonpersistence and nonadherence with oral 5-aminosalicylic acid therapy in patients with ulcerative colitis. J Manag Care Pharmacy. 2012;18(9):701.

82. Altobelli E, et al. Current psoriasis treatments in an Italian population and their association with socio-demographical and clinical features. J Eur Acad Dermatol Venereol. 2012;26(8):976-82.

83. Bhosle MJ, et al. Medication adherence and health care costs associated with biologics in Medicaid-enrolled patients with psoriasis. J Dermatol Treat. 2006;17(5):294-301.

84. Chan SA, et al. Factors affecting adherence to treatment of psoriasis: comparing biologic therapy to other modalities. J Dermatol Treatment. 2013;24(1):64-9.

85. Chastek B, et al. Psoriasis treatment patterns with etanercept and adalimumab in a United States health plan population. J Dermatolog Treat. 2013;24(1):25-33.

86. Clemmensen A, et al. Responses to ustekinumab in the anti-TNF agent-naïve versus anti-TNF agent-exposed patients with psoriasis vulgaris. J Eur Acad Dermatol Venereol. 2011;25(9):1037-40.

87. Esposito $M$, et al. Survival rate of antitumour necrosis factor- $\alpha$ treatments for psoriasis in routine dermatological practice: a multicentre observational study. Br J Dermatol. 2013;169(3):666-72.

88. Gniadecki R, et al. Comparison of drug survival rates for adalimumab, etanercept and infliximab in patients with psoriasis vulgaris. Br J Dermatol. 2011;164(5):1091-6.
89. Gokdemir G, Arı S, Köşlü A. Adherence to treatment in patients with psoriasis vulgaris: turkish experience. J Eur Acad Dermatol Venereology. 2008;22(3):330-5.

90. Richards HL, et al. Patients with psoriasis and their compliance with medication. J Am Acad Dermatol. 1999;41(4):581-3.

91. Umezawa Y, et al. Drug survival rates in patients with psoriasis after treatment with biologics. J Dermatol. 2013;40(12):1008-13.

92. Zaghloul S, Goodfield M, Jeremey D. Objective assessment of compliance with psoriasis treatment. Arch Dermatol. 2004;140(4):408-14.

93. De Korte J, et al. Quality of life in patients with psoriasis: a systematic literature review.J Investigative Dermatol Symposium Proc. 2004. Nature Publishing Group.

94. Coleman CI, et al. Dosing frequency and medication adherence in chronic disease. J Manag Care Pharm. 2012;18(7):527-39.

95. Saini SD, et al. Effect of medication dosing frequency on adherence in chronic diseases. Am J Manag Care. 2009;15(6):e22-33.

96. Kardas P, et al. 3 report on the determinants of patient non-adherence with short-term therapies and treatments for chronic diseases in Europe. Ascertaining barriers for compliance: policies for safe, effective and cost-effective use of medicines in Europe Final Report of the ABC Project (Deliverable 7.1), 2012. p. 40.

97. DiMatteo MR, Lepper HS, Croghan TW. Depression is a risk factor for noncompliance with medical treatment: meta-analysis of the effects of anxiety and depression on patient adherence. Arch Intern Med. 2000;160(14):2101-7.

98. Arnett PA, Higginson CI, Voss WD, Bender WI, Wurst JM, Tippin JM. Depression in multiple sclerosis: relationship to working memory capacity. Neuropsychology. 1999;13(4):11.

99. Gadkari AS, McHorney CA. Unintentional non-adherence to chronic prescription medications: how unintentional is it really? BMC Health Services Res. 2012;12(1):98.

100. Van den Bemt B, et al. Making the rheumatologist aware of patients' non-adherence does not improve medication adherence in patients with rheumatoid arthritis. Scand J Rheumatol. 2011;40(3):192-6. 